\title{
Effect of microvesicles from Moringa oleifera containing miRNA on proliferation and apoptosis in tumor cell lines
}

\author{
Marina Potestà', Valentina Roglia', Marialaura Fanelli', Elisa Pietrobono', Angelo Gismondi', Simone Vumbaca (1) , \\ Rick Gildas Nguedia Tsangueu ${ }^{1}$, Antonella Canini ${ }^{1}$, Vittorio Colizzi ${ }^{1}$, Sandro Grelli ${ }^{2}$, Antonella Minutolo ${ }^{1}$ and \\ Carla Montesano ${ }^{1}$
}

\begin{abstract}
Human microvesicles are key mediators of cell-cell communication. Exosomes function as microRNA transporters, playing a crucial role in physiological and pathological processes. Plant microvesicles (MVs) display similar features to mammalian exosomes, and these MVs might enhance plant microRNA delivery in mammals. Considering that plant microRNAs have been newly identified as bioactive constituents in medicinal plants, and that their potential role as regulators in mammals has been underlined, in this study, we characterized MVs purified from Moringa oleifera seeds aqueous extract (MOES MVs) and used flow cytometry methods to quantify the ability to deliver their content to host cells. The microRNAs present in MOES MVs were characterized, and through a bioinformatic analysis, specific human apoptosis-related target genes of plant miRNAs were identified. In tumor cell lines, MOES MVs treatment reduced viability, increased apoptosis levels associated with a decrease in B-cell lymphoma 2 protein expression and reduced mitochondrial membrane potential. Interestingly, the effects observed with MOES MVs treatment were comparable to those observed with MOES treatment and transfection with the pool of small RNAs isolated from MOES, used as a control. These results highlight the role of microRNAs transported by MOES MVs as natural bioactive plant compounds that counteract tumorigenesis.
\end{abstract}

\section{Introduction}

Cells are in constant communication with each other. This communication is mainly mediated by extracellular vesicles, which are small transporters made up of a lipidic membrane that act as carriers of molecules ${ }^{1}$. In this context, extracellular vesicles have recently been suggested to be able to transfer their cargo into exogenous recipient cells, mediating communication between unrelated species ${ }^{1-5}$.

Correspondence: Carla Montesano (montesano@uniroma2.it)

'Department of Biology, University of Rome 'Tor Vergata', via della Ricerca Scientifica 1, 00133 Rome, Italy

${ }^{2}$ Department of Experimental Medicine and Surgery, University of Rome "Tor Vergata", Via Montpellier 1, 00133 Rome, Italy

These authors contributed equally: Marina Potestà, Valentina Roglia, Antonella Minutolo, Carla Montesano

Edited by M. V. Niklison Chirou
Plant microvesicles (MVs) are a heterogeneous class of vesicles that play unique functions, as protective compartments, for intercellular transport of multiple materials, contributing to plant growth and development, defense responses, and plant-microbe symbiosis ${ }^{6}$.

Plant MVs have been classified as "exosome-like" because their morphology and density are similar to those of mammalian exosomes .

Several in vitro and in vivo studies have suggested that edible plant-derived MVs can accumulate in mammalian cells, and have important functional effects on recipient cells. These plant-derived MVs seem to participate in intestinal tissue homeostasis in healthy subjects and have anti-inflammatory effects in tumor cells ${ }^{8}$.

\section{(c) The Author(s) 2020}

(c) (i) Open Access This article is licensed under a Creative Commons Attribution 4.0 International License, which permits use, sharing, adaptation, distribution and reproduction cc) in any medium or format, as long as you give appropriate credit to the original author(s) and the source, provide a link to the Creative Commons license, and indicate if changes were made. The images or other third party material in this article are included in the article's Creative Commons license, unless indicated otherwise in a credit line to the material. If material is not included in the article's Creative Commons license and your intended use is not permitted by statutory regulation or exceeds the permitted use, you will need to obtain permission directly from the copyright holder. To view a copy of this license, visit http://creativecommons.org/licenses/by/4.0/. 
Small RNA and microRNA (sRNA, miRNA), together with lipids, secondary metabolites, and proteins, have been shown to be present in plant extracellular vesicles ${ }^{8}$.

MVs appear to enhance sRNA stability; in fact, "microvesicles and its content in sRNAs", have been shown to be resistant to pasteurization, homogenization and ultrasonic treatments ${ }^{6,9}$.

These MVs may mediate plant-animal communication, having a role in cross-kingdom regulation processes, by delivering plant sRNAs and miRNAs to mammalian systems ${ }^{7,10-13}$.

MiRNAs are short stretches of noncoding RNAs that play an essential role in gene regulation; they regulate protein expression at the post-transcriptional level in animals and plants. More than four hundred conserved miRNAs have been identified from plants, and in silico and in vitro functional validation have highlighted the possible role of plant miRNAs as regulators of protein expression in mammals ${ }^{11,14-21}$.

According to these data, sRNAs and miRNAs present in medicinal plants might act as bioactive compounds in mammals.

In many African communities, Moringa oleifera Lam. (MO) is widely used for preparations of traditional remedies. The benefits of MO-based preparations are scientifically documented ${ }^{22,23}$. These studies demonstrated that MO bioactivity depends on the presence of different classes of plant secondary metabolites ${ }^{24,25}$. In 2016, the miRNome of MO was sequenced, showing the presence of several conserved miRNAs ${ }^{26-30}$. Potestà et al. ${ }^{31}$ reported that $\mathrm{MO}$ seed aqueous extract (MOES), is able to differentially regulate proliferation and apoptosis in healthy and cancer cells and that this ability is associated with the presence of miRNAs.

Therefore, in the current work the MVs present in the MOES previously studied ${ }^{31}$ were extracted and characterized and the ability of these vesicles to enter human tumor cells and induce proapoptotic and antiproliferative effects were investigated.

\section{Results}

Characterization and delivery of MVs extracted from MOES

Plant Mvs fulfill two roles: miRNA protection and transport of into recipient cells ${ }^{14,17,32}$.

In the present research, size and content of MOES MVs were characterized; moreover, their role in cell host was investigated.

Using the Megamix-Plus SSC (Biocytex, France) standard as a reference in flow cytometry analysis, we identified a population of 100-500 nm MOES MVs (Fig. 1a), as described in the Materials and Methods.

The dimensions of MOES MVs were comparable to those of the Megamix control. Specifically, 59\% of the MOES MVs analyzed were between 240 and $500 \mathrm{~nm}$ in size, $3.65 \%$ were between 200 and $240 \mathrm{~nm}$, and only $2 \%$ were $<200 \mathrm{~nm}$ (Fig. 1b, c).

The contents of MOES MVs, analyzed by spectrophotometer, showed an absence of DNA (Fig. 1f, i) but presence of RNA, proteins, and lipids: the MOES MVs present in $10 \mathrm{mg}$ of MOES contained $1.63 \pm 0.03 \mu \mathrm{g} / \mu \mathrm{l}$ of protein and $7.9 \pm 0.75 \mathrm{ng} / \mu \mathrm{l}$ of RNA (Table 1a).

Using specific probes to identify the presence of lipids, RNA and DNA, Flow cytometry analysis was performed. Among the MOES MVs, a significant number of SYTO RNA and BODIPY (BPI) positive MVs (Fig. 1d, e, Table 1b) with a significant increase in Mean Fluorescence Intensity (MFI) (Fig. 1g, h) was observed.

Table $1 \mathrm{~b}$ also contains data on the quantification of RNA, DNA, and lipids determined by flow cytometry analysis of a $50 \mu \mathrm{l}$ MVs sample extracted from $10 \mathrm{mg}$ of MOES. The results are reported as the number of events measured in $50 \mu \mathrm{l}$ of sample that were positive for the probes used to detect the presence of lipids, DNA, and RNA. As reported in Table 1b, there are a $\%$ of $42.49 \pm 3.65$ positive cells for the RNA probe and $20.63 \pm 0.58$ for the BPI probe. The characterization of MOES MVs showed that lipids, proteins and RNA are present, whereas DNA was not found.

Host cell internalization of SYTO RNA-labelled MOES MVs was observed in Jurkat cells and PBMCs from HDs by flow cytometry (Fig. 2a-d) and HeLa cells through microscopy (Fig. 2e, f). In both cell lines and in PBMCs from HDs, a significant increase in fluorescence intensity was observed 30 minutes after treatment (Fig. 2b, d, f), indicating their ability to naturally enter into the cell without needing other carrier.

\section{Cytotoxic effect of MOES and MOES MVs}

To obtain information about the effects of MOES MVs on cell viability, Jurkat and HeLa cells, and PBMCs from HDs were treated with MOES at a concentration ranging from 0 to $50 \mathrm{mg} / \mathrm{ml}$ and with the number of MOES MVs purified from each investigated MOES concentration. Seventy-two hours after treatment, cell viability was analyzed using a trypan blue assay.

MOES and MOES MVs treatment at $1 \mathrm{mg} / \mathrm{ml}$ induced a significant reduction in Jurkat cells viability that was dose dependent (Fig. 3a).

HeLa cells were more resistant to MOES and MOES MVs treatment, showing a significant reduction in viability, starting at $5 \mathrm{mg} / \mathrm{ml}$ for both MOES and MOES MVs (Fig. 4a).

The treatments had different cytotoxic effects: both MOES and MOES MVs significantly increased the number of trypan blue-positive Jurkat cells. However, starting at $5 \mathrm{mg} / \mathrm{ml}$, MOES was significantly more toxic than MOES MVs treatment at the same concentrations (Fig. 3b).

In HeLa cells, $10 \mathrm{mg} / \mathrm{ml}$ MOES and MOES MVs were required to significantly increase the number of trypan bluepositive cells, confirming that HeLa cells were more resistant to 


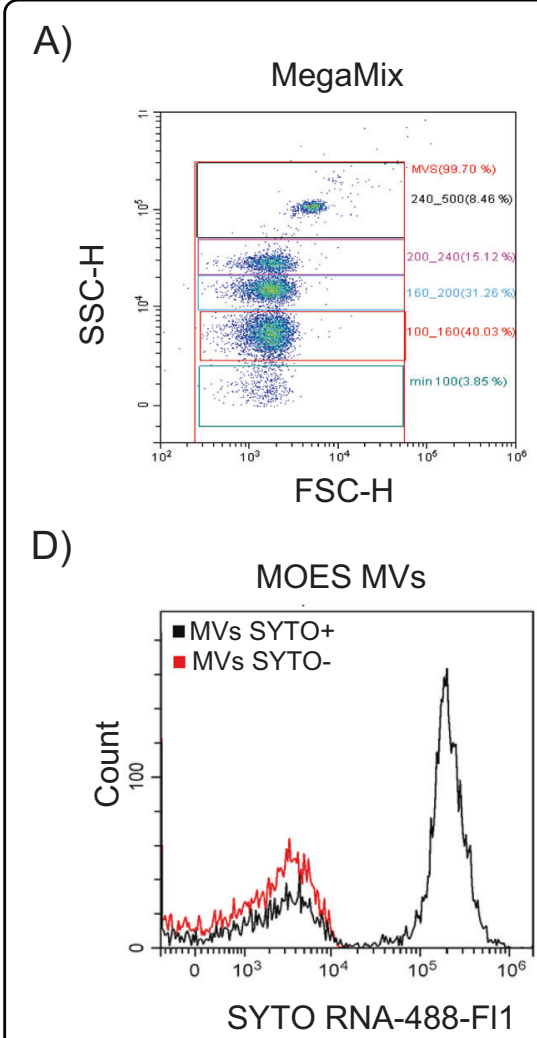

B)

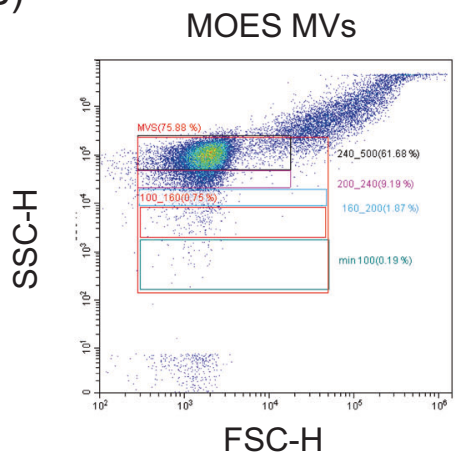

E)

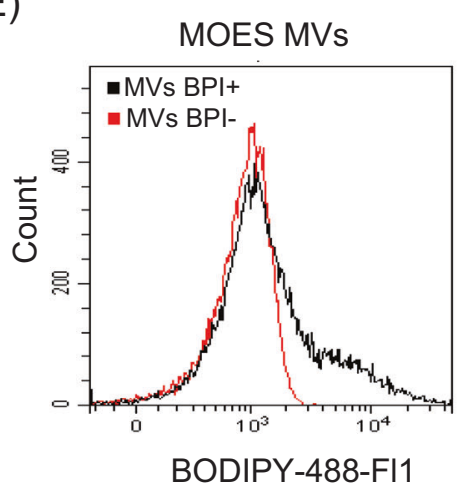

H)

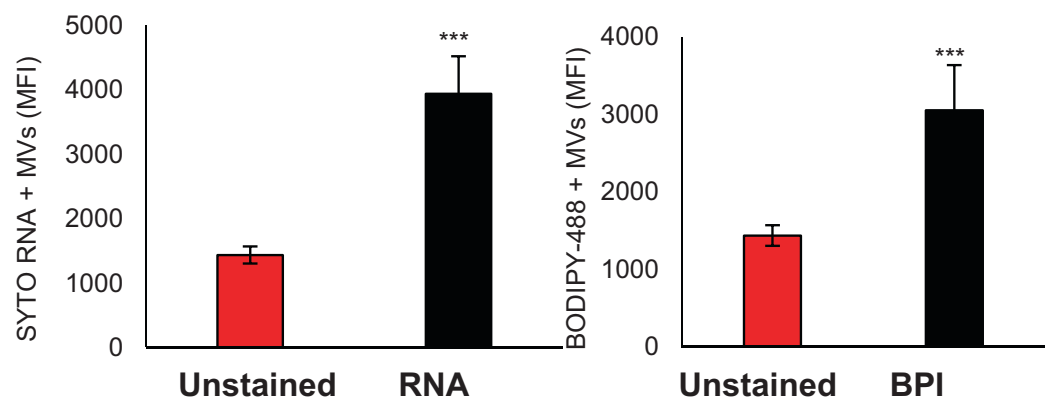

C)

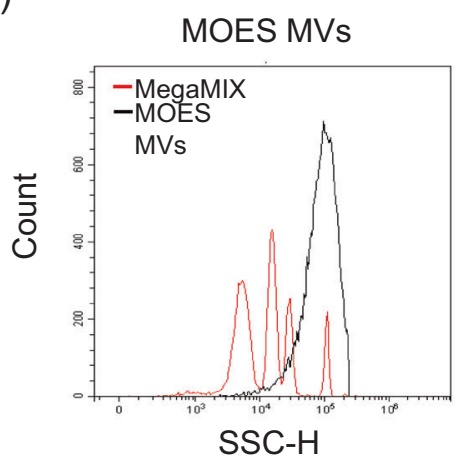

F)

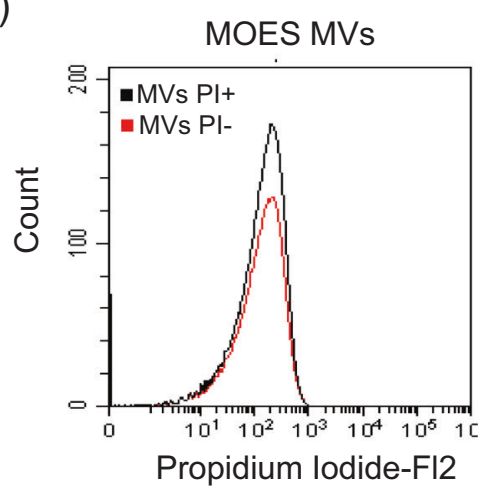

I)

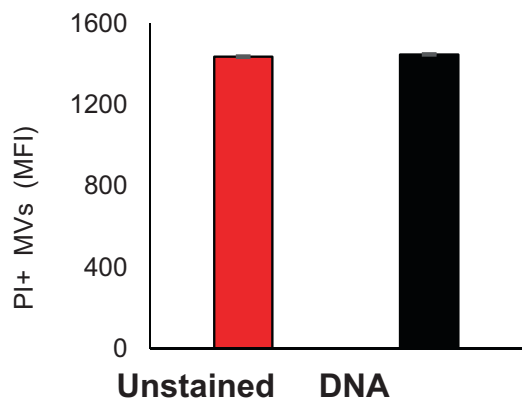

Fig. 1 Characterization of MVs extracted from MOES. A representative pseudo-dot plot (FSC-H vs SSC-H, a) of the standardized fluorescent (FITCA) nanosized particles of different sizes (100-160 nm, 160-200 nm, 200-240 nm, 240-500 nm) from the Megamix-Plus SSC kit used as a control for the analysis of the dimensions of the vesicles in the sample. The FSC-H and SSC-H parameters of MOES MVs extracted from $10 \mathrm{mg}$ MOES is shown on b. In a representative histogram of the SSC-H parameter, the Megamix reference particles show four peaks corresponding to the dimensions described above (b, red line) compared with the control, the MVs extracted from MOES (black line, $\mathbf{c}$ ) show a greater presence of microvesicles with a diameter between 240 and $500 \mathrm{~nm}$. The MVs marked with probes for RNA, lipids, and DNA were analyzed by flow cytometry. On d, a representative overlay histogram of the SYTO RNA marked MVs compared with the unmarked MVs. g represents the mean \pm SD of the SYTO RNA Mean Fluorescence Intensity (MFI) of three independent measurements made using three different samples of MVs. For the analysis of lipid content, a BODIPY probe was used: e shows a representative histogram overlay of the BODIPY-positive MVs compared with the unmarked MVs. $\mathbf{h}$ represents the mean \pm SD of the BODIPY MFI of three independent measurements made using three different samples of MVs. For the analysis of DNA content, a propidium iodide (PI) probe was used: $\mathbf{f}$ shows a representative histogram overlay of the PI-positive MVs compared with the unmarked MVs. $\mathbf{i}$ represent the mean \pm SD of the PI MFI of three independent measurements made using three different samples of MVs. Data are reported as the mean of three different experiments \pm SD. $t$ test was used; symbols indicate significant differences: ${ }^{* *} p<0.001$.

this type of treatment. Moreover, there was no difference in the induction of cell death between MOES and MOES MV-treated HeLa cells, at any of the analyzed concentrations (Fig. 4b).
Considering these results, the concentrations of MOES and MOES MVs able to reduce cell viability to $50 \%$ of that at time 0 (EC50) after $72 \mathrm{~h}$ of treatment were calculated (Table 2). 
Table 1a Characterization of MVs derived from $10 \mathrm{mg}$ of MOES.

\begin{tabular}{ll}
\hline & Concentration \\
\hline Total RNA & $7.9 \pm 0.75 \mathrm{ng} / \mu \mathrm{l}$ \\
Protein & $1.63 \pm 0.03 \mu \mathrm{g} / \mu \mathrm{l}$ \\
DNA & undetectable \\
\hline
\end{tabular}

Mean \pm SD of three independent measurements. The content of RNA and DNA was dosed using a spectrophotometer (Pharmacia Biotech Ultrospec 3000), for protein quantification BIORAD method was used.

Jurkat cells were more susceptible to MOES treatment than to MOES MVs treatment. Indeed, $13.63 \pm 0.024 \mathrm{mg} /$ $\mathrm{ml}$ MOES was enough to reduce Jurkat cell viability by $50 \%$ (EC50), whereas the number of MOES MVs corresponding to $3.57 \pm 0.004 \mathrm{mg} / \mathrm{ml}$ MOES was necessary to reduce viability by $50 \%$.

Moreover, MOES induced 50\% cell death (LD50) more effectively than MOES MVs. Specifically, the concentration of MOES that induced 50\% Jurkat cell death (LD50) was $8.42 \pm 0.026 \mathrm{mg} / \mathrm{ml}$, whereas the number of MOES MVs corresponding to $22.91 \pm 0.001 \mathrm{mg} / \mathrm{ml} \mathrm{MOES} \mathrm{was}$ necessary to induce $50 \%$ cell death (Table 2 ).

In the adhesive HeLa cell line, treatment with MOES MVs was less toxic than treatment with the MOES preparation, as previously observed in Jurkat cells. Indeed, $22.31 \pm 0.03 \mathrm{mg} / \mathrm{ml}$ MOES was required to inhibit cell viability by $50 \%$, which is $10 \mathrm{mg}$ more than that in Jurkat cells. MOES MVs were also less toxic for HeLa cells than the Jurkat cells, requiring the number of MOES MVs corresponding to $45.63 \pm 0.025 \mathrm{mg} / \mathrm{ml}$ MOES to reach a $50 \%$ inhibition of cell growth. These data confirmed that adhesive cells exhibited a greater resistance to these treatments than suspension cells and that both cells lines exhibited a greater resistance to MOES MVs than to MOES (Table 2).

Previously, MOES treatment was shown to have these toxic effects on different tumor lines but not on PBMCs from HDs, suggesting that MOES treatment may specifically target cancer cells ${ }^{31}$.

The effects of MOES MVs treatment on cell viability were therefore investigated on PBMCs from HDs stimulated with IL2 (Fig. 4e).

Neither MOES nor MOES MVs treatment affected the viability/death of PBMCs from HDs at any analyzed concentration (Fig. 4e), confirming the ability of these extracts to have toxic effects specifically on cancer cells and not on healthy ones.

Effect of MOES and MOES MVs on cell cycle and apoptosis

To understand whether the effects on viability/mortality were associated with an induction of apoptosis and cell
Table 1b Characterization of Lipids, DNA, and RNA contents with BODIPY fluorescent probe (Thermo Fisher Scientific, USA), propidium iodide, and SYTO RNA-staining methods, respectively.

\begin{tabular}{lll}
\hline & No. of events & \% of positive events \\
\hline Total events in MVs gate & $19224.5 \pm 3536$ & - \\
BPI + (Lipids) & $3844.5 \pm 365$ & $20.63 \pm 0.58$ \\
PI + (DNA) & 0 & 0 \\
SYTO RNA + & $7762.65 \pm 135$ & $42.49 \pm 3.65$ \\
\hline
\end{tabular}

Mean \pm SD of three independent measurements. Flow cytometry analysis was performed via Cytoflex (Beckman Coulter, USA) and CytExpert 2.2 software (Beckman Coulter, USA).

cycle perturbation, we stained the cells with PI after $72 \mathrm{~h}$ of treatment and performed flow cytometry analysis. In Jurkat cells, there was a significant increase in the percentage of apoptotic cells starting at $1 \mathrm{mg} / \mathrm{ml} \mathrm{MOES}$ (Fig. 3c).

Treatment with higher MOES concentrations resulted in a significant increase in apoptosis in a concentrationdependent manner. Treatment with MOES MVs caused a significant increase in apoptosis starting at $10 \mathrm{mg} / \mathrm{ml}$, proving that this type of treatment is less toxic (Fig. 3d). The cell cycle analysis showed a significant reduction in the number of events and in the percentage of cells in G2 phase related to a significant increase in the cells undergoing to apoptosis (Table S2).

In the HeLa cell line, higher concentrations of MOES and MOES MVs were needed to induce apoptosis than in the Jurkat cell line. Indeed, $5 \mathrm{mg} / \mathrm{ml}$ MOES was needed to observe a significant induction of apoptosis (Fig. 4c).

Treatment at higher concentrations increased the percentage of hypodiploid cells in a dose-dependent manner. In addition, MOES MVs were less toxic than MOES, and a concentration of $20 \mathrm{mg} / \mathrm{ml}$ was necessary to obtain a significant increase in apoptosis (Fig. 4d). As for the Jurkat, the cell cycle analysis showed a significant reduction of the HeLa cells in phase G2 and an increase in the number and percentage of apoptotic cells (Table S2).

Apoptosis in PBMCs appeared to be comparable to that in the untreated control at all concentrations tested, confirming that only cells of tumor origin were susceptible to the treatments (Fig. 4f).

Considering these data, we chose the following concentrations for subsequent studies: $1 \mathrm{mg} / \mathrm{ml}$ MOES and $10 \mathrm{mg} / \mathrm{ml}$ MOES MVs for the Jurkat cell line and $5 \mathrm{mg} / \mathrm{ml}$ MOES and $20 \mathrm{mg} / \mathrm{ml}$ MOES MVs for the HeLa cell line.

To confirm that the treatments induced apoptosis at these concentrations, the Jurkat and HeLa cells were stained early after treatment (48 hours) and assessed using the annexin V/PI assay to distinguish early apoptotic cells (annexin $\mathrm{V}+/ \mathrm{PI}-$ ) and late apoptotic/necrotic cells 
A)

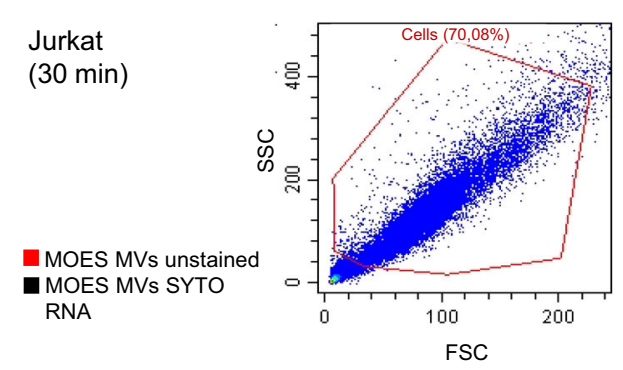

C)

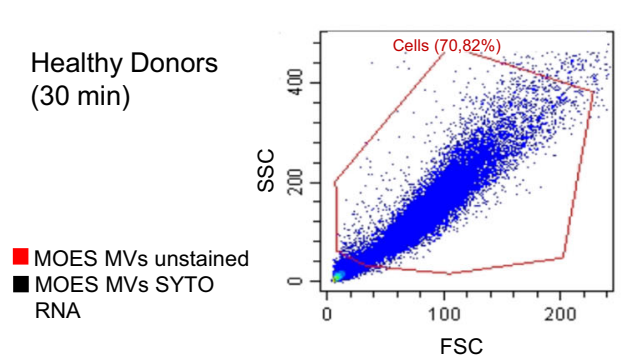

E)

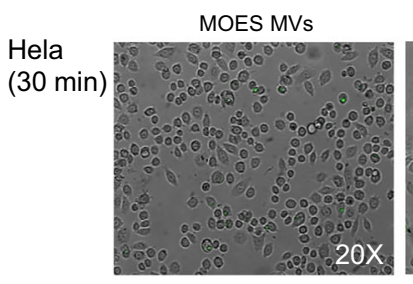

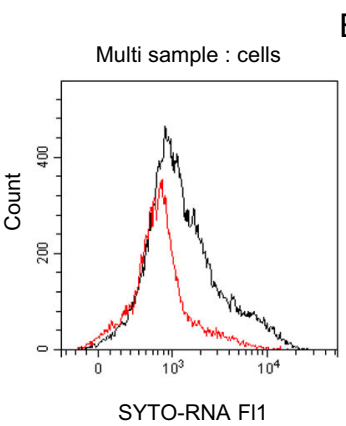

B)

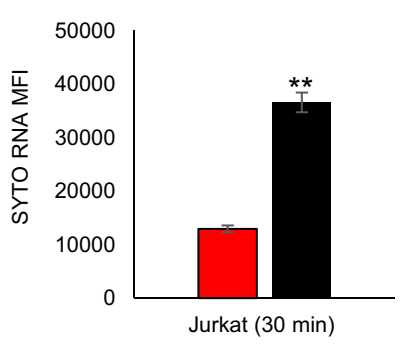

D)
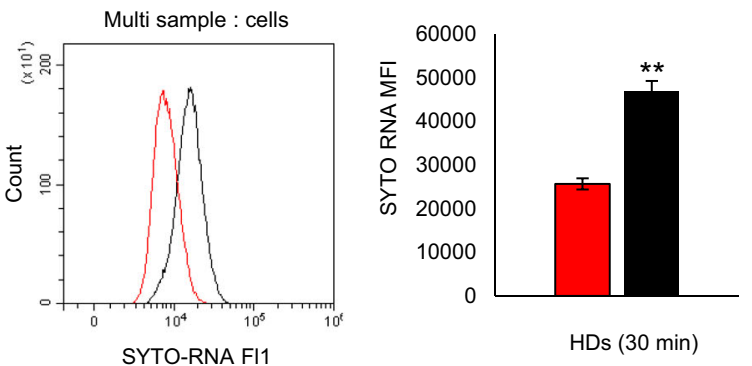

F)

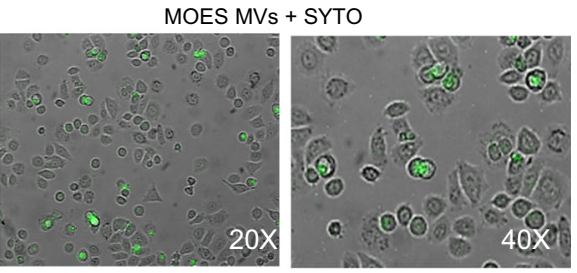

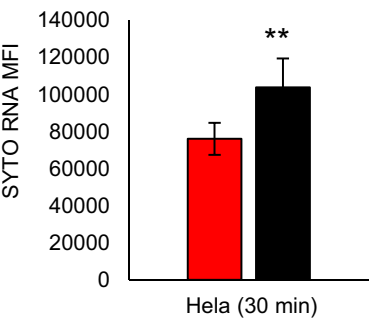

Fig. 2 Delivery of MOES MVs to Jurkat cells, HeLa cells, and PBMCs from healthy donors. The presence of stained MOES MVs in Jurkat cells, HeLa cells and PBMCs from healthy donors was investigated after 30 minutes of treatment with MOES MVs stained with SYTO RNA and analyzed by flow cytometry analysis or microscopy. Jurkat and Healthy donors were gated for cells (a and $\mathbf{c}$, left side). Representative overlay histograms of Jurkat cells (a, right side) and PBMCs (c, right side) labelled with SYTO RNA-stained MOES MVs (black line) compared with unlabeled cells (red line) are presented. The MFI of SYTO RNA-positive Jurkat cells and PBMCs is reported in $\mathbf{b}$ and $\mathbf{d}$, respectively, representing the mean \pm SD of the MFI of three independent measurements made using three different samples of MVs. In HeLa cells, delivery was assessed by fluorescence microscopy analysis of RNA-positive HeLa cells. e shows a representative delivery experiment analyzed by fluorescence microscopy: the left picture shows HeLa cells treated only with MVs; the middle and left pictures of e shows the cells at two magnifications $(\times 20$ and $\times 40)$ after incubation for 30 minutes with MVs marked with the RNA probe. The MFI of SYTO RNA-positive HeLa cells is represented by the mean \pm SD of three independent measurements of three different samples of MVs $\mathbf{f}$. Data are reported as the mean of three different experiments \pm SD. $t$ test was used; symbols indicate significant differences: ${ }^{* *} p<0.001$ stained vs unstained cells.

(annexin $\mathrm{V}+/ \mathrm{PI}+)^{33}$. The results shown in Fig. 3e are representative of two experiments performed on Jurkat cells. Table 3 reports the data obtained on Jurkat and HeLa cells.

After 48 hours of treatment with MOES, Jurkat and HeLa cells showed a significant increase in the percentage of annexin V-positive cells compared with untreated cells. Conversely, the levels of late apoptotic/necrotic cells (annexin $\mathrm{V}+/ \mathrm{PI}+$ ) in the sample exposed to the treatment were similar to those detected in the control sample. Treatment with MOES MVs significantly increased annexin V-positive cells in both tumor cell lines.

In addition, in the sample treated with MOES MVs, the levels of late apoptotic/necrotic cells (annexin $\mathrm{V}+/ \mathrm{PI}+$ ) were similar to those detected in the control sample. Clearly, these experiments asserted that MOES and MOES MVs were able to specifically induce cell death by apoptosis. 


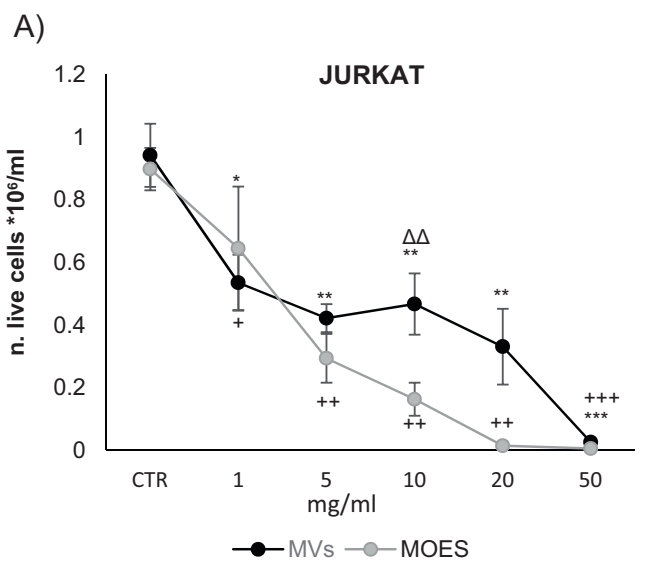

B)
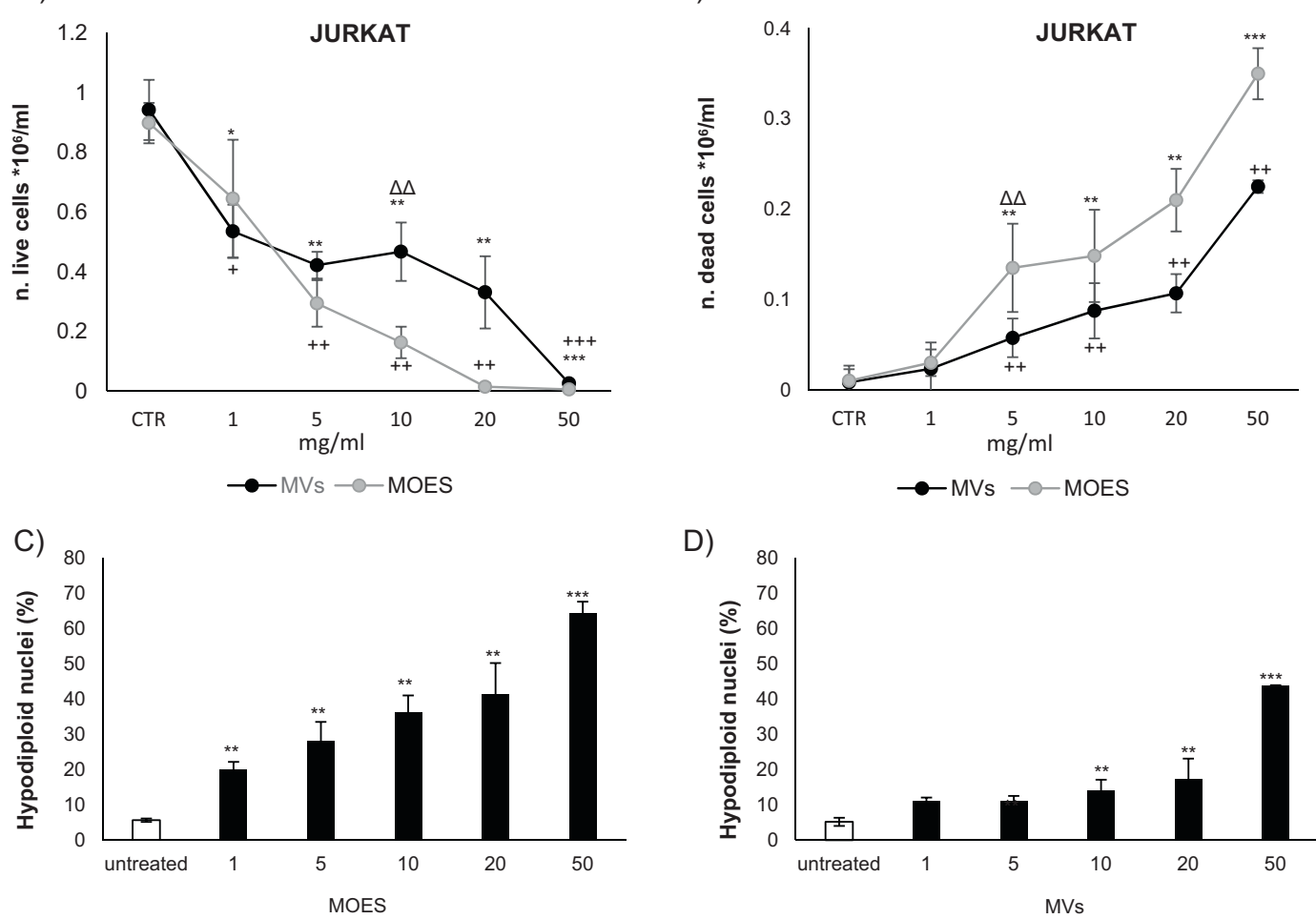

D)

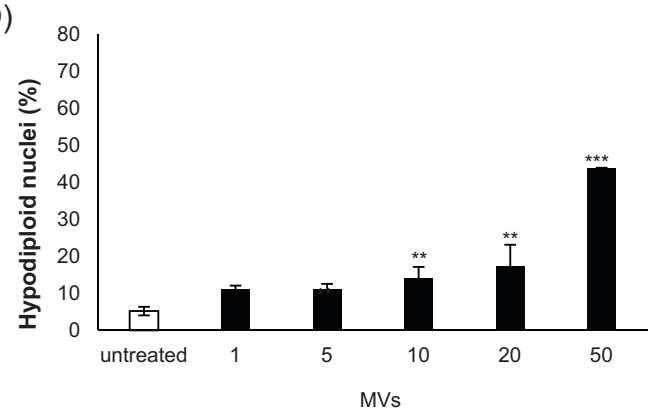

E)

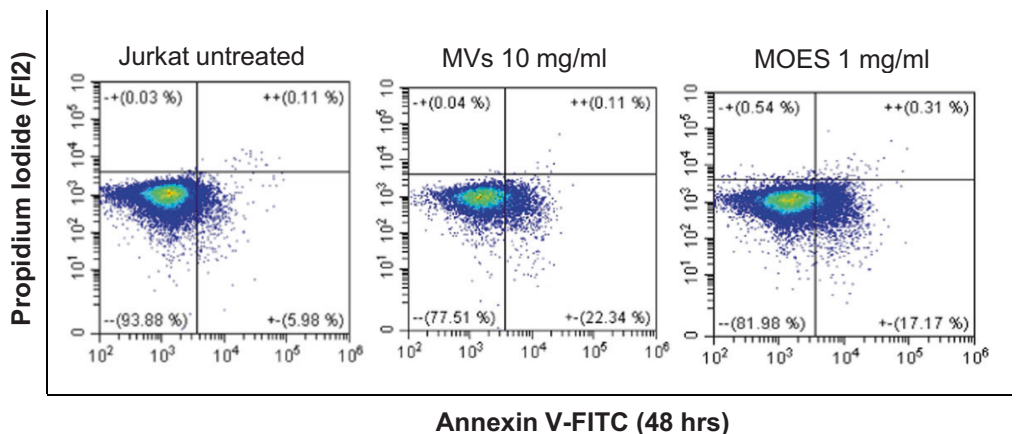

Fig. 3 Cytotoxic effect of MOES and MOES MVs on Jurkat cells. Cell viability and mortality analyzed by the Trypan blue exclusion assay in Jurkat cells $\mathbf{a}$ and $\mathbf{b}$ after $72 \mathrm{~h}$ treatment with MOES at concentrations ranging from 0 to $50 \mathrm{mg} / \mathrm{ml}$ or MVs purified from the corresponding concentrations of MOES. Control cells were incubated for the same time with an equivalent volume of distilled water. The results are expressed as number of trypan blue-negative $\mathbf{a}$ or trypan blue-positive $\mathbf{b}$ cells. Effects of MOES $\mathbf{c}$ and MOES MVs $\mathbf{d}$ on apoptosis in Jurkat cells. Apoptosis was evaluated as the percentage of hypodiploid nuclei by flow cytometry analysis after $72 \mathrm{~h}$ of incubation. e shows a representative dot plot of the percentages of annexin V-, propidium iodide-, and annexin V/propidium iodide-positive Jurkat cells treated with MOES and MOES MVs after 48 hours of incubation. Data are reported as the mean of three different experiments \pm SD. Symbols indicate significant differences in viability/death and apoptosis: ${ }^{*} p<$ $0.05{ }^{* *} p<0.01,{ }^{* * *} p<0.001$ MOES-treated vs untreated cells. ${ }^{+} p<0.05,{ }^{++} p<0.01,{ }^{+++} p<0.001$ MOES MVs-treated vs untreated cells. ${ }^{\Delta} p<0.05$, ${ }^{\Delta \Delta} p<0.01,{ }^{\Delta \Delta} p<0.001$ comparisons of the concentrations of the different treatments (MOES vs MOES MVs) able to significantly modify cell viability or death. $t$ test was used for Apoptosis and Trypan blu assay analysis; ANOVA and a Bonferroni multiple comparison test for annexin V/propidium iodide assay were used.

miRNA expression profile of MOES MVs and MOES

The previous results demonstrated the antiproliferative and proapoptotic effects of MOES on cancer cells. Considering the results published in Potestà et $\mathrm{al}^{31}$, we investigated the possible role of miRNAs carried by MOES MVs in these specific mechanisms.

Starting from the characterization of the M. oleifera miRNome ${ }^{29}$, the presence of specific miRNAs belonging 
A)

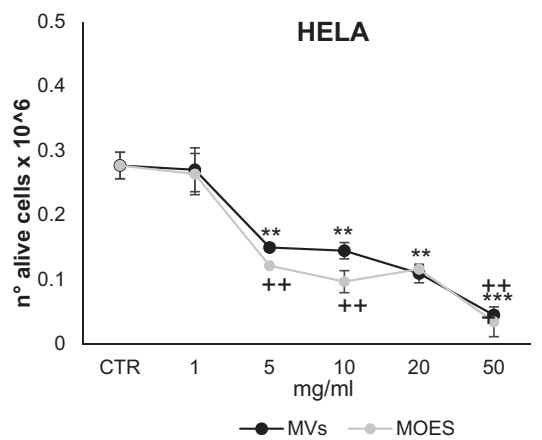

C)

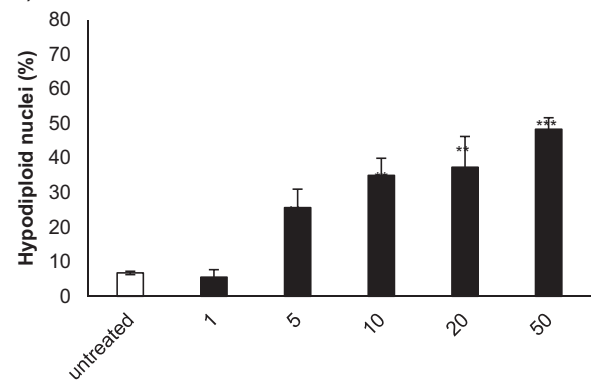

MOES

E)

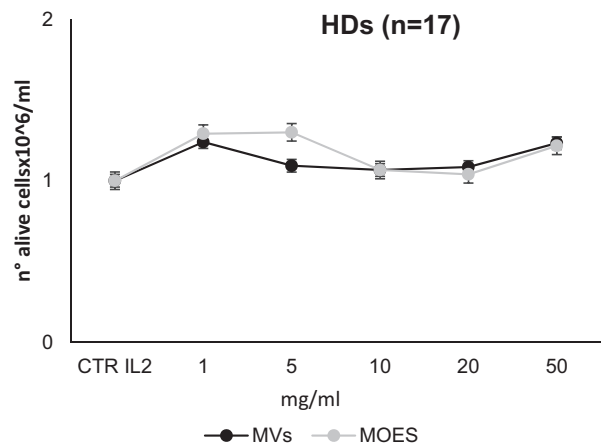

B)

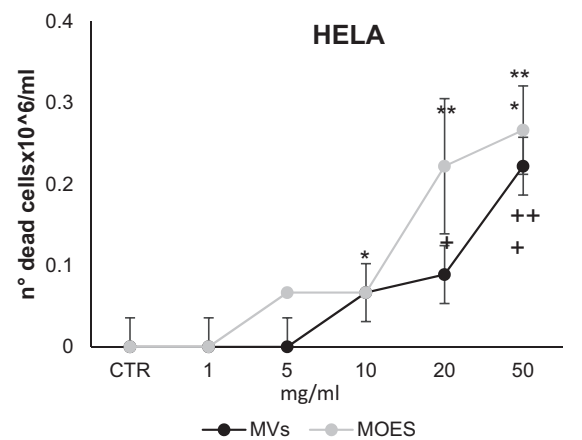

D)

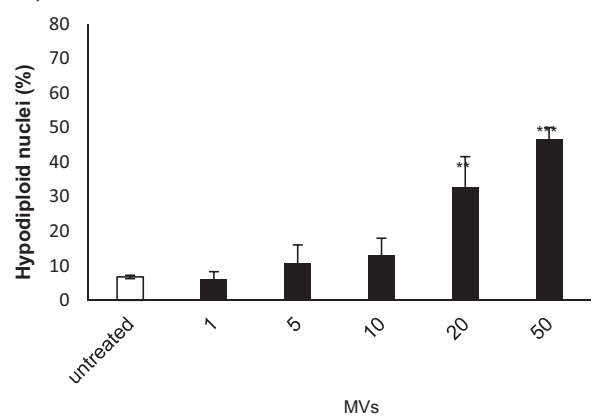

F)

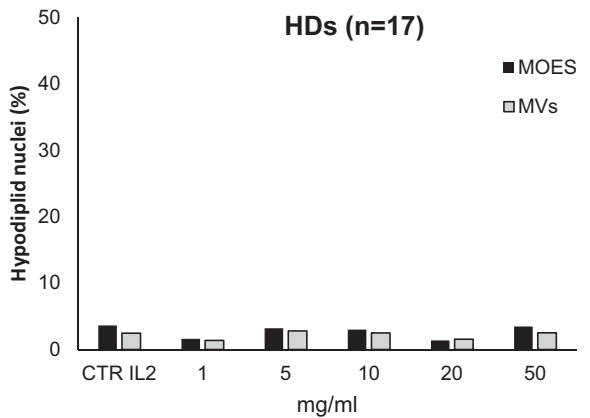

Fig. 4 Cytotoxic and apoptotic effects of MOES and MOES MVs on HeLa cells and PBMCs from HDs. Cell viability and mortality analyzed by the Trypan blue exclusion assay in HeLa cells (a and $\mathbf{b}$ ) and PBMCs e after $72 \mathrm{~h}$ treatment with MOES at concentrations ranging from 0 to $50 \mathrm{mg} / \mathrm{ml}$ or MOES MVs purified from the corresponding concentrations of MOES. Control cells were incubated for the same time with an equivalent volume of distilled water. The results are expressed as number of Trypan blue-negative $\mathbf{a}$ and $\mathbf{e}$ or trypan blue-positive $\mathbf{b}$ cells. Effects of MOES $\mathbf{c}$ and MOES MVs d on apoptosis in HeLa cells and PBMCs f. Apoptosis was evaluated as the percentage of hypodiploid nuclei by flow cytometry analysis after 72 hours of incubation. Data are reported as the mean of three different experiments \pm SD. Symbols indicate significant differences in viability/death and apoptosis: $t$ test was used for Apoptosis and Trypan blu assay analysis; ${ }^{*} p<0.05,{ }^{* *} p<0.01,{ }^{* * *} p<0.001$ MOES-treated vs untreated cells. ${ }^{+} p<0.05$, ${ }^{++} p<0.01,{ }^{+++} p<0.001$ MOES MVs-treated vs untreated cells.

to 20 conserved families of plant miRNAs was evaluated in the $\mathrm{mol}_{\text {-sR }}$ pool ${ }^{31}$. Here, by qPCR analysis, we detected and quantified the presence of these miRNAs (miRs) in the pool of sRNAs extracted from the MOES MVs. Among the $21 \mathrm{~mol}$-miRs belonging to the 20 most conserved plant miRNA families, 19 were found in MOES MVs. The comparison between the miRNAs present in MOES MVs and those identified in the MOES highlighted that conserved plant miRNAs were present at high concentrations in both samples (Table 4). The amount of miRNAs in MOES and MOES MVs preparations were comparable, except that miR396a and miR396c, which belong to a specific plant miRNA family (miRNA modulators for growth-regulating factors) ${ }^{34}$. In particular, these miRNAs were highly expressed in MOES MVs, compared with MOES (Table 4). 
Table 2 Cytotoxic effect of MOES and MOES MVs treatments on Jurkat and HeLa cell lines and PBMCs from Healthy donors $(n=17)$.

\begin{tabular}{lll}
\hline & MOES FW $\mathbf{~ g} / \mathbf{m l}$ & MOES MVs $\mathbf{~ g} / \mathbf{m l}$ \\
\hline Jurkat & & \\
EC50 & $13.63 \pm 0.024^{\mathrm{a}}$ & $31.57 \pm 0.004$ \\
LD50 & $8.42 \pm 0.026$ & $22.91 \pm 0.011$ \\
HeLa & & \\
EC50 & $22.31 \pm 0.03^{\mathrm{a}}$ & $45.63 \pm 0.025$ \\
LD50 & $11.63 \pm 0.01$ & $12.31 \pm 0.01$ \\
HDs & & \\
EC50 & $>100$ & $>100$ \\
LD50 & $>100$ & $>100$ \\
\hline
\end{tabular}

Mean \pm SD of three independent measurements. Trypan blue assay results from the different concentrations of extracts were compared using ANOVA and a Bonferroni significant difference test as a multiple comparison test. ${ }^{a}$ MOES vs MOES MVs, $p<0.01$.

\section{Computational prediction of genes targeted by mol-miRs} recovered from MOES and MOES MVs

Following the characterization of the miRs content in MOES MVs, a bioinformatic analysis was performed to identify the possible human genes targeted by the miRs present in the mol-sR pool and involved in the apoptosis mechanism $^{16,20,35}$.

The results show that among the 19 most conserved mol-miRs found in MOES MVs, 12 could potentially bind human mRNAs involved in apoptosis (Table 5).

Among them, four (mol-miR160h; mol-miR482b; molmiR166; mol-miR 159c) showed the possibility to modulate the expression of $>10$ genes with antiapoptotic activity, which has been shown to be deregulated in tumorigenic mechanisms.

\section{Effect of miRs contained in MOES MVs on proliferation and apoptosis BCL2 mediated}

In previous works, the ability of plant miRNAs ${ }^{16}$ to induce cell death by BCL2-mediated apoptosis in tumor cells has been demonstrated. Moreover, the antiproliferative and proapoptotic properties of MOES were correlated to BCL2 modulation ${ }^{31}$.

For these reasons, MOES MVs treatments were carried out on Jurkat and HeLa cell lines to understand whether the effects of MVs-carried miRs on viability and apoptosis were BCL2-mediated.

Jurkat and HeLa cell lines were treated with the concentrations of MOES MVs and MOES previously chosen. In addition, a purified $m o l$-sR pool was used as a positive control in transfection experiments, to determine the ability of the MVs to enter the cell naturally and the efficacy of the treatment directly mediated by the miRs transported by the MVs.
After $72 \mathrm{~h}$, compared with the control, all treatments induced a significant reduction of proliferation in both Jurkat and HeLa cells (Fig. 5a, b). Specifically, Jurkat cells showed a significant decrease in the number of alive cells in presence of $1 \mathrm{mg} / \mathrm{ml} \mathrm{MOES}$, the number of MVs corresponding to $10 \mathrm{mg} / \mathrm{ml}$ (Fig. 5a) and $1 \mu \mathrm{g} / \mathrm{ml} \mathrm{mol}$-sRs. A reduction in cell proliferation was evident in HeLa cells treated with $5 \mathrm{mg} / \mathrm{ml}$ MOES, the MOES MVs number corresponding to $20 \mathrm{mg} / \mathrm{ml} \mathrm{MOES}$ and $5 \mu \mathrm{g} / \mathrm{ml} \mathrm{mol}$-sRs (Fig. 5b).

Treatment with MOES, MOES MVs, and mol-sRs induced a significant increase in apoptosis in both analyzed cell lines. All treatments determined a significative increase in the percentage of hypodiploid nuclei in Jurkat and HeLa cells respect to untreated cells (Fig. 5c, d). The proapoptotic effect was further investigated by evaluating the expression of BCL2 protein through flow cytometry analysis. Treatment with MOES MVs induced a significant decrease in the percentage (red line) and MFI (black barr) in BCL2-positive cells (Fig. 5e, g for Jurkat and $\mathrm{F}, \mathrm{h}$ for HeLa).

Notably, all the effects of MOES MVs were comparable to those of MOES and mol-miR. Taken together, these data suggest that MOES MVs induce a reduction in cell growth and an increase in apoptosis in tumor cell lines that is associated with a significant downregulation of BCL2 protein expression. The transfection of tumor cells with the purified mol-sR pool had a comparable effect to MOES and MOES MVs treatments, suggesting that this proapoptotic and response is due to the presence of the miRs in the analyzed extracts.

\section{Effects of MOES and MOES MVs on mitochondrial membrane potential}

Mitochondria play an important role in BCL2-mediated apoptosis: the collapse of the mitochondrial membrane potential (MMP) mirrors the early events leading to apoptotic cell death ${ }^{36,37}$. MMP collapse was detected with the cationic dye JC-1: MMP collapse is associated with an increase in the accumulation of monomeric JC-1, low-red fluorescent forms in the cytoplasm.

Thus, the percentage of monomeric JC-1-positive cells in the presence of MOES, MOES MVs and mol-sRs was investigated to reflect the number of cells undergoing apoptosis. Cells treated with CCCP exhibited high levels of both red (FL2) and green (FL1) fluorescence, whereas samples treated with MOES, MOES MVs, and mol-miRs showed a significant progressive decrease in high-red fluorescent aggregates and an accumulation of cells positive for the monomeric forms of $\mathrm{JC}-1$ in the lower right (LR) quadrant of the cytogram (Fig. 6a, c). At the same time, the percentage of monomeric JC-1-positive cells in the UR+LR quadrant of the cytogram increased in both cell lines with all treatments. Consistent with the 
Table 3 Percentage of Annexin V (Anx V), propidium iodide (PI), and annexin V/propidium iodide (Anx V/PI)-positive cells in Jurkat and HeLa cells treated with MOES and MOES MVs after 48 hours of incubation.

\begin{tabular}{|c|c|c|c|c|}
\hline Jurkat & Anx V $+\mathrm{PI}-$ & Anx $\mathbf{V}+\mathbf{P I}+$ & $\begin{array}{l}\text { Anx V - PI } \\
+\end{array}$ & $\begin{array}{l}\text { Anx V - PI } \\
-\end{array}$ \\
\hline Untreated & $4.80 \pm 2.40$ & $0.18 \pm 0.03$ & $0.51 \pm 0.20$ & $92.56 \pm 0.14$ \\
\hline $\mathrm{MVs} 10 \mathrm{mg} / \mathrm{ml}$ & $21.20 \pm 1.07^{* * *}$ & $0.77 \pm 0.15^{*}$ & $0.81 \pm 0.29 \mathrm{~ns}$ & $88.65 \pm 0.36$ \\
\hline MOES1 mg/ml & $18.92 \pm 0.13^{* * *}$ & $0.25 \pm 0.14^{*}$ & $0.08 \pm 0.15 \mathrm{~ns}$ & $82.61 \pm 0.24^{*}$ \\
\hline Hela & $A n x V+P I-$ & $A n x V+P l+$ & $A n \times V-P I+$ & Anx $V-P I-$ \\
\hline Untreated & $10.80 \pm 2.40$ & $0.16 \pm 0.41$ & $0.52 \pm 0.20$ & $89.73 \pm 0.14$ \\
\hline MVs $20 \mathrm{mg} / \mathrm{ml}$ & $23.16 \pm 1.12^{* *}$ & $0.14 \pm 0.26$ & $0.18 \pm 0.9 \mathrm{~ns}$ & $83.16 \pm 0.61^{*}$ \\
\hline MOES $5 \mathrm{mg} / \mathrm{ml}$ & $21.80 \pm 0.74^{* * *}$ & $0.25 \pm 0.10$ & $0.61 \pm 0.05 \mathrm{~ns}$ & $81.71 \pm 0.24^{*}$ \\
\hline
\end{tabular}

Mean \pm SD of two independent measurements, ${ }^{*} p<0.05,{ }^{* *} p<0.01,{ }^{* * *} p<0.001$; MOES or MOES MVs vs untreated (ANOVA-Bonferroni multiple comparison test).

MMP collapse induced by the treatments, values of red/ green mean fluorescence ratios progressively and significantly decreased following treatment in all cell lines assayed (Fig. 6b, d and Table 6).

Mitochondrial function was also determined by the MitoTracker red assay. During MMP collapse, MitoTracker Red is retained in mitochondria, and the MFI is directly correlated with mitochondrial polarization status. Viable untreated cells were highly fluorescent, whereas in all treatment conditions, there was a significant decrease in fluorescence indicative of apoptotic cells (Fig. 6e-h).

Taken together, these results clearly showed the ability of the mol-miRs present in MOES MVs to regulate mitochondria-mediated antiproliferative and proapoptotic mechanisms in cancer cells.

\section{Discussion}

In recent years, several studies have reported beneficial effects of MO on human health ${ }^{38-40}$. The Food and Agriculture Organization of the United Nations (FAO) recommends the consumption of $\mathrm{MO}$, especially for pregnant women, nursing mothers and young children, because it is rich in antioxidants and other essential micronutrients that are lacking in malnourished populations living in underdeveloped and developing countries $^{41}$.

The whole plant is used, from roots to seeds ${ }^{40}$. Literature has reported that MO strengthens and stimulates the immune system owing to the presence of antiradical molecules, minerals, and essential amino acids that are also able to maintain proper blood circulation and fluidization, thereby benefiting the circulatory and cardiovascular system ${ }^{38,40-44}$. MO also has a high antiinflammatory, which is often at the basis of pathologies such as cancer, cardiovascular diseases, dementia, and arthritis ${ }^{40,43}$.

Zhang $^{18}$ and others works ${ }^{16,20,45}$ introduced the crosskingdom interaction concept in which miRNAs present in
Table 4 Characterization of microRNA from MOES and MOES MVs.

\begin{tabular}{|c|c|c|c|}
\hline & MOES MVs & MOES & $\begin{array}{l}\text { MOES MVs vs } \\
\text { MOES } p<0.001\end{array}$ \\
\hline mol-miR $159 \mathrm{c}$ & 417752.12 & 137806.81 & \\
\hline mol-miR 156a & 1262.69 & 763.93 & \\
\hline mol-miR 858 & 30.32 & 934.01 & \\
\hline mol-miR 395a & 302.81 & 267.29 & \\
\hline mol-miR 162a & 64.10 & 655.88 & \\
\hline mol-miR 171d & 1736.89 & 712.77 & \\
\hline mol-miR 2118a & 1025.63 & 447.98 & \\
\hline mol-miR 482b & 1011.50 & 341.87 & \\
\hline mol-miR 396c & 272614606.23 & 327.94 & $* * *$ \\
\hline mol-miR 396a & 49546768.16 & 10.91 & $* * *$ \\
\hline mol-miR 168a & 98.52 & 71.13 & \\
\hline mol-miR 397-5p & 80.02 & 6.26 & \\
\hline mol-miR $167-5 p$ & 827.31 & 943.77 & \\
\hline mol-miR 166i & 561.17 & 642.38 & \\
\hline mol-miR 398b & 7.79 & 3.28 & \\
\hline mol-miR $160 \mathrm{~h}$ & 9.33 & 4.37 & \\
\hline mol-miR 393a & 7.12 & 20.64 & \\
\hline mol-miR 159a & 57940.62 & 12096.38 & \\
\hline mol-miR 858b & 1 & 1 & \\
\hline
\end{tabular}

Relative RT-qPCR expression analysis of the most conserved mol-miRNAs contained in the pool of sRNAs extracted from MOES and MOES MVs. The analysis was carried out in three independent biological experiments and expressed as fold change with respect to $\mathrm{mol}$-miR858b expression, previously normalized with a housekeeping gene ( $5 \mathrm{~S}$ rRNA). The relative expression of $\mathrm{mol}-$ miRs was quantified by the $2^{-\Delta \Delta \mathrm{Ct}}$ method and reported as $2^{-\Delta \Delta \mathrm{ct}} \times 10^{5}$.

plant extracts, introduced by diet, are able to control gene expression in human cells. Different studies ${ }^{21,26,46,47}$ have confirmed this hypothesis: plant sRNAs containing 
Table 5 Bioinformatics analysis of possible human mRNA targets of miRs contained in the mol-sR pool and involved in the apoptosis process.

\begin{tabular}{|c|c|c|c|c|}
\hline miRNA & No. of target & $\begin{array}{l}\text { mRNA } \\
\text { Antiapoptotic }\end{array}$ & No. of target & $\begin{array}{l}\text { mRNA } \\
\text { Proapoptotic }\end{array}$ \\
\hline mol-mir160h & 21 & $\begin{array}{l}\text { bfar, bnip3l, ripk2, igf1r, cd40lg, bcl-2110, nol3, bnip3, tnf, bcl-2l2, bcl-2l1, xiap, } \\
\text { nfkb1, hrk, akt1, bag3, mcl1, dapk1, naip, birc3, birc5 }\end{array}$ & 4 & ripk2, tnf, hrk, akt1 \\
\hline mol-mir482b & 17 & $\begin{array}{l}\text { mcl1, bfar, igf1r, hrk, braf, bcl-2l2, bcl-211, dapk1, akt1, nol3, bcl2, birc3, cd27, il10, } \\
\text { xiap, bax, bag3 }\end{array}$ & 3 & hrk, akt1, bax \\
\hline mol-mir166 & 14 & bax, braf, mcl1, cd27, bnip3, bcl-212, bag1, nfkb1, cflar, igf1r, hrk, birc5, bcl2, akt1 & 2 & hrk, akt1 \\
\hline mol-mir 159c & 9 & cd40lg, il10, birc6, naip, dapk1, tnf, bcl-2|10, bcl-2|1, bnip3l & 2 & tnf, bnip3l \\
\hline mol-mir2118a & 5 & tnf, bax, akt1, bcl-212, bnip3 & 4 & tnf, bax, akt1, bnip3 \\
\hline mol-mir167f-3p & 5 & tnf, akt1, bcl-212, cd40lg, bnip3l & 3 & tnf, akt1, bnip3l \\
\hline mol-mir156e & 4 & bag1, bag3, cflar, bcl-211 & & \\
\hline mol-mir395d & 4 & bag1, akt1, il10, bnip3l & 2 & akt1, bnip3l \\
\hline mol-mir393a & 3 & bnip2, bnip3l, dapk1 & 1 & bnipl3 \\
\hline mol-mir397a & 2 & dapk1, ripk2 & 1 & ripk2 \\
\hline mol-mir858b & 1 & nol3 & & \\
\hline mol-mir396a & 1 & il10 & & \\
\hline
\end{tabular}

miRNAs may be considered a new class of micronutrients responsible for the medical properties of plants.

MiRNAs are short nucleotide sequences that interact with mRNA to regulate gene expression at the posttranscriptional level.

How exogenous plant miRNAs ingested with food persist in the mammalian intestine for several hours and are present in body fluids affecting gene expression in humans has been widely discussed ${ }^{48,49}$. Plant miRNAs present a methyl group linked to a 2' hydroxyl on their 3' ends that increases their stability during cooking processes, protects them from enzymatic digestion, improve their bioavailability and promotes their regulatory activity in humans ${ }^{46}$. Owing to these characteristics, the use of plant miRNAs in therapeutic practices is of interest. Furthermore, the ability of plant miRNAs to regulate human mRNA stability and translation and their presence in biological fluids has been demonstrated ${ }^{16,20,35}$. What remains to be understood is how these miRNAs can cross the intestinal barrier. Considering the instability of naked RNA, the ability of plant miRNAs to resist pepsin, pancreatin, and bile can be attributed to the presence of protein complexes and lipid vesicles that encapsulate them, protecting them from degradation. The presence of MVs in plants has raised many questions about their biological relevance and potential health benefits. Recent data have demonstrated that MVs from food might have unsuspected functions in mouse digestive tract and thus might participate in whole-body homeostasis ${ }^{8}$. Moreover, in the literature, plant extracellular vesicles, which carry bioactive molecules, such as proteins, mRNAs, and miRNAs, have been shown to act on biological mechanisms in recipient cells. A recent study demonstrated the ability of stained vesicles to cross the intestinal barrier, showing their presence in other regions of the body, such as the liver, spleen, and lymphatic circulation, in mice with colitis $^{8}$.

For several years, we had studied the effects of natural substances, specifically the bioactive component of the seed aqueous extract of $M O$ obtained using a traditional African procedure ${ }^{31}$ : the antiproliferative and proapoptotic MOES properties on tumor cells were demonstrated, and these effects could be attributed to the presence of miRs contained in the aqueous extract.

In this work, we demonstrated that MOES MVs (carrying miRs) are able to enter human cells and modulate activities related to viability and apoptosis in tumor cell lines.

After extracting MVs from aqueous plant extracts, we characterized the bioactive components (proteins, DNA, RNA, and lipids) of MOES MVs and assessed the dimension of MOES MVs through flow cytometry analysis.

MOES MVs were found to contain proteins, RNA, and lipids but not DNA.

As expected, RNA characterization revealed the presence of miRs belonging to the most conserved plant miRNA family. 
A)

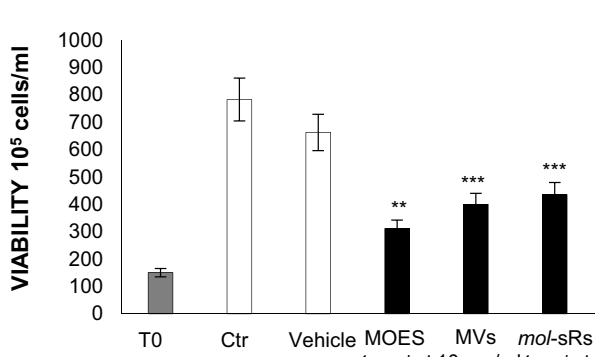

C)

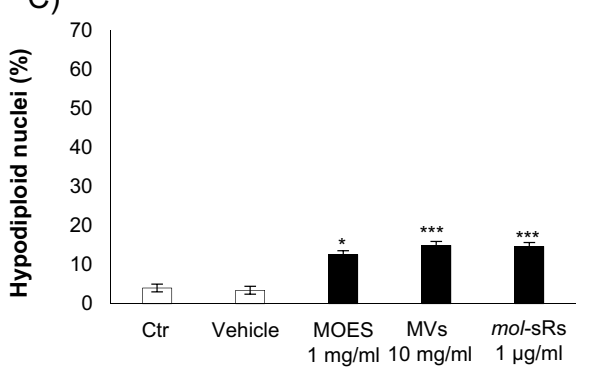

E)

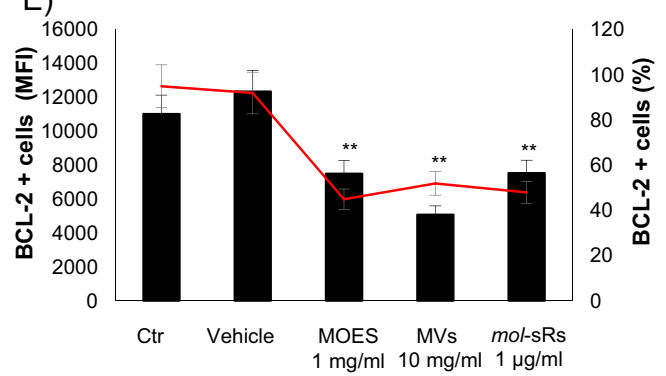

G)

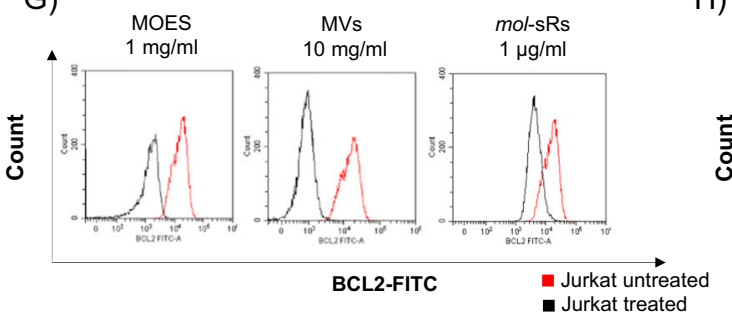

B)

HELA
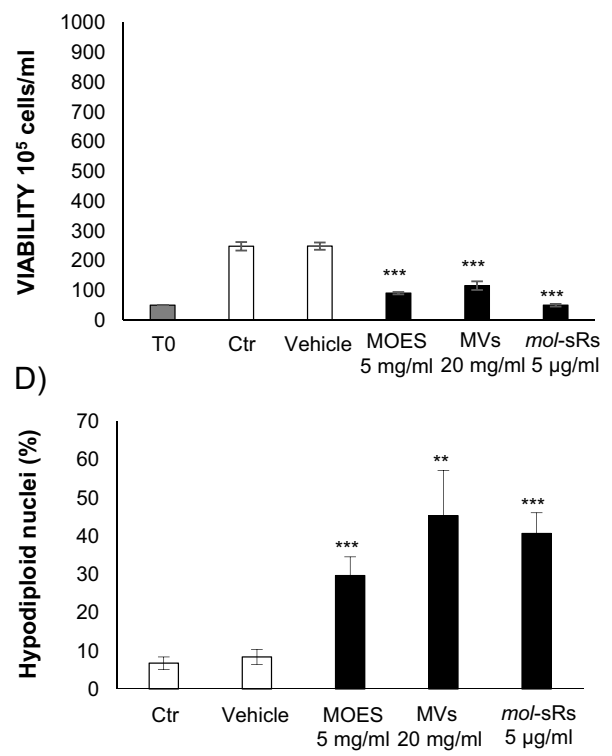

F)

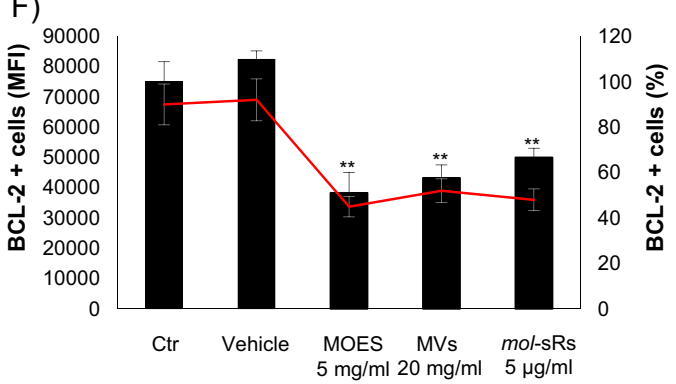

H)

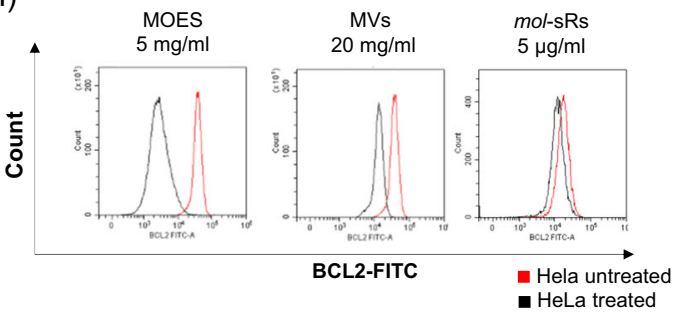

Fig. 5 Characterization of the BCL2-mediated antiproliferative and proapoptotic effects of MOES and MOES MVs. a and $\mathbf{b}$ show the viability of Jurkat and HeLa cells, respectively, measured with the Trypan blue exclusion assay. Jurkat cells were treated for 72 hours with $1 \mathrm{mg} / \mathrm{ml}$ MOES or MVs corresponding to $10 \mathrm{mg}$ MOES or were transfected with $1 \mathrm{\mu g} / \mathrm{ml}$ mol-sRs as a positive control. HeLa cells were treated with $5 \mathrm{mg} / \mathrm{ml}$ MOES or MVs corresponding to $20 \mathrm{mg} \mathrm{MOES} \mathrm{or} \mathrm{were} \mathrm{transfected} \mathrm{with} 5 \mathrm{\mu g} / \mathrm{ml} \mathrm{mol}-\mathrm{sRs}$. The data are reported as the mean \pm SD of the number of live cells $\times$ $10^{6} / \mathrm{ml}$. Apoptosis was evaluated as the percentage of hypodiploid nuclei by flow cytometry analysis $(\mathbf{c}, \mathbf{d})$ and reported as the percentage of apoptotic cells. BCL2 intracellular protein expression in Jurkat and HeLa cells was evaluated by flow cytometry analysis of live cell gates after 72 hours of treatment. e, $\mathbf{f}$ report the MFI (black barr) and percentage (red line) of BCL2-positive Jurkat and HeLa cells from three independent biological experiments. One representative overlay histogram of BCL2 protein expression in Jurkat $\mathbf{g}$ and HeLa $\mathbf{h}$ cells treated with MOES, MVs or mol-sRs compared with untreated cells (red line). Data are reported as the mean \pm SD of three independent experiments performed. Symbols indicate significant differences: ${ }^{*} p<0.05,{ }^{* *} p<0.01,{ }^{* * *} p<0.001$ for all treated $v$ s untreated cells. Comparisons between treated and untreated cells were all conducted using $t$ test.

Bioinformatic analysis is widely used to evaluate the possible interactions between miRNAs and their mRNA targets $^{10,14,20,35}$. Considering our previous works in which the miRNome of $\mathrm{MO}^{29}$ was sequenced and the proapoptotic and antiproliferative effects of MOES were evaluated $^{31}$, here, we identified the possible human genes 

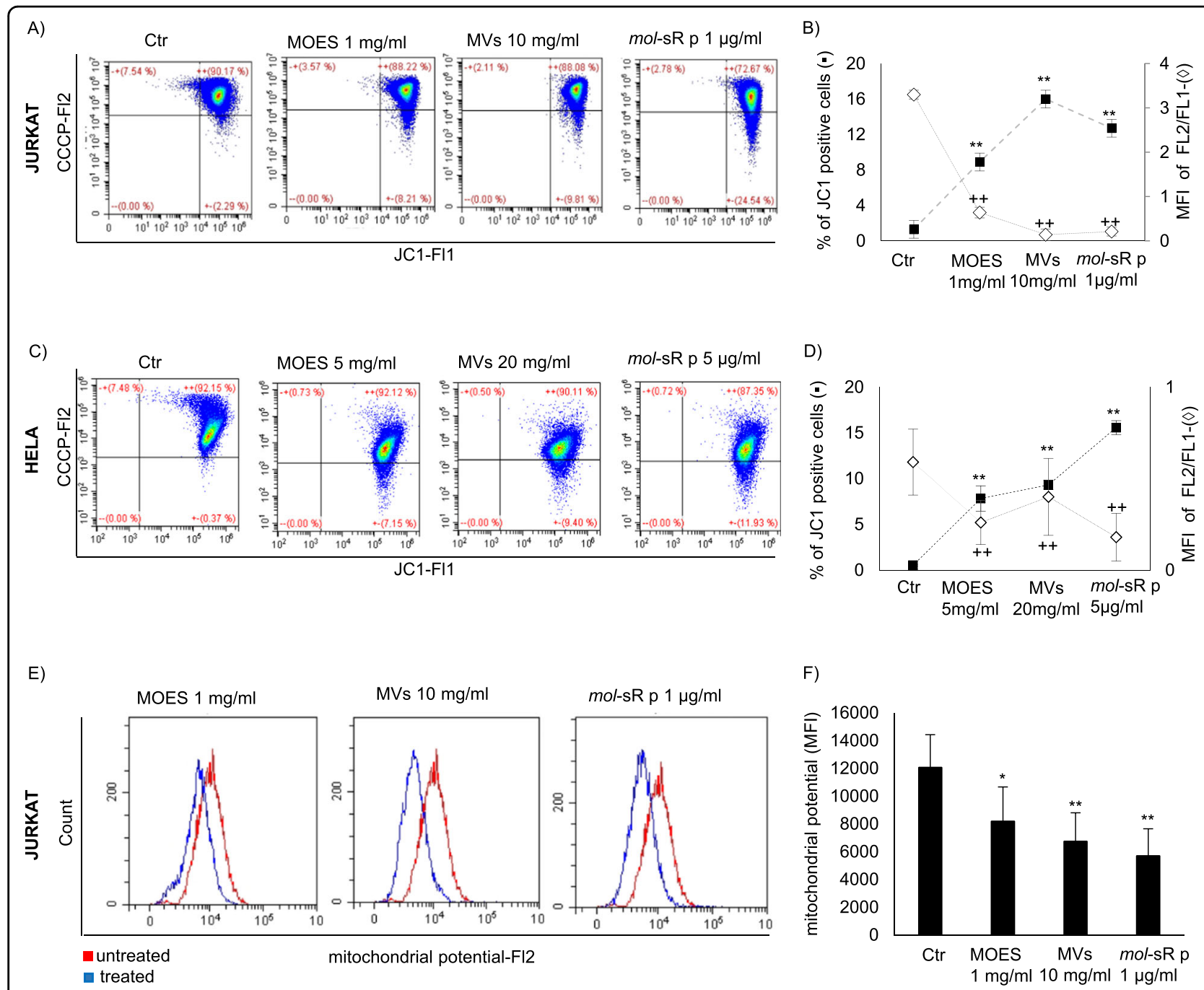

G)

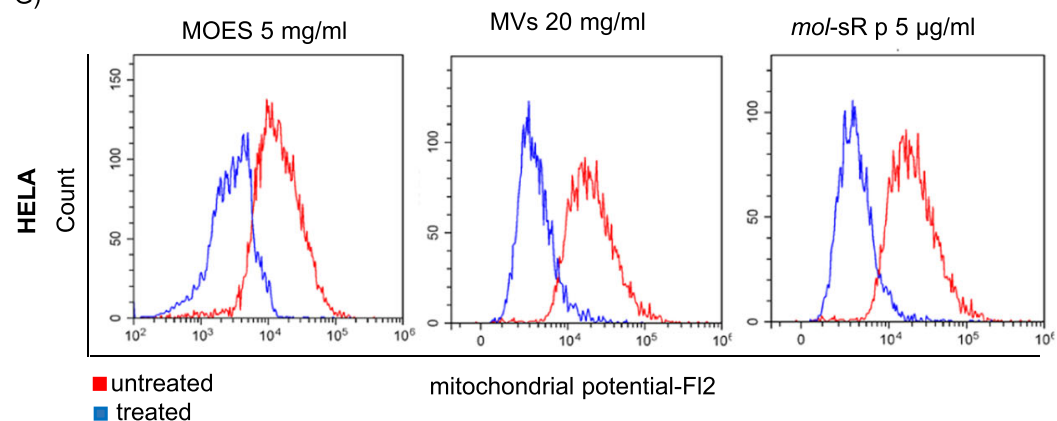

H)

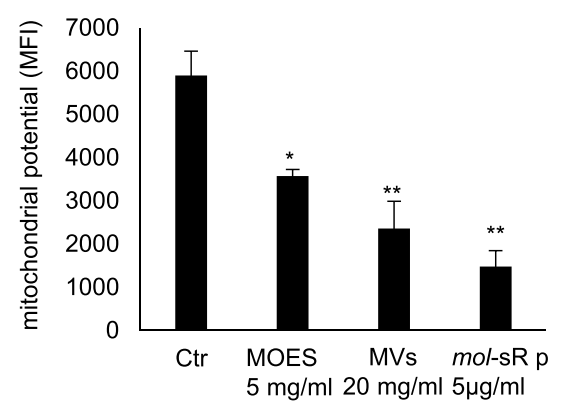

Fig. 6 Effects of MOES and MOES MVs on MMP in Jurkat and HeLa cells. The mitochondrial potential in the Jurkat and HeLa cell lines was evaluated by flow cytometry analysis using JC-1 and MitoTracker Red FM probes on live cell gates after $72 \mathrm{~h}$ of treatment with MOES, MVs, or mol-sRs. $\mathbf{a}$ and $\mathbf{c}$ show a representative pseudo-dot plot of JC-1-stained Jurkat and HeLa cells. The percentage of monomeric JC-1-positive cells and the JC-1 $\mathrm{red} / \mathrm{green}$ mean fluorescence ratios are represented in $\mathbf{b}$ for Jurkat cells and $\mathbf{d}$ for HeLa cells. The results of three independent experiments performed for each cell line are expressed as the mean \pm SD of the percentage of cells accumulated in the LR quadrant (black square), as well as the red/green mean fluorescence intensity ratio (white rhombus). Representative overlay histograms of MMP expression in Jurkat and HeLa cells stained with MitoTracker Red FM (e and $\mathbf{g}$ ) $72 \mathrm{~h}$ after treatment compared with MMP expression in untreated cells. The MFI of MitoTracker Red FM-positive cells from three independent biological experiments is reported in $\mathbf{f}$ and $\mathbf{h}$. Data are reported as the mean \pm SD of three independent experiments performed. ANOVA and a Bonferroni multiple comparison test were used; symbols indicate significant differences: ${ }^{*} p<0.05$, ${ }^{* *} p<0.01$; all treated vs untreated cells. 
Table 6 Detection of MMP in Jurkat and HeLa cells.

\begin{tabular}{lll}
\hline Jurkat & \% of JC-1-positive cells & MFI (FL2/FL1) \\
\hline CTR & $1.3 \pm 1.41$ & $3.3 \pm 0.75$ \\
MOES1 mg/ml & $8.9 \pm 0.95^{*}$ & $0.64 \pm 0.70^{*}$ \\
MVS $10 \mathrm{mg} / \mathrm{ml}$ & $12.70 \pm 4.07^{* *}$ & $0.21 \pm 0.00^{* *}$ \\
mol-sR $1 \mu \mathrm{g} / \mathrm{ml}$ & $26.00 \pm 2.06^{* *}$ & $0.14 \pm 0.12^{* *}$ \\
HeLa & $\%$ of JC-1-positive cells & MFI (FL2/FL1) \\
CTR & $0.53 \pm 0.10$ & $0.59 \pm 0.48$ \\
MOES $5 \mathrm{mg} / \mathrm{ml}$ & $7.82 \pm 1.38^{* *}$ & $0.26 \pm 0.22^{* *}$ \\
MVS $20 \mathrm{mg} / \mathrm{ml}$ & $9.31 \pm 0.53^{* *}$ & $0.40 \pm 0.41^{* *}$ \\
mol-sR $5 \mu \mathrm{g} / \mathrm{ml}$ & $15.57 \pm 0.76^{* *}$ & $0.18 \pm 0.13^{* *}$ \\
\hline
\end{tabular}

ANOVA and a Bonferroni multiple comparison test was used. Mean \pm SD of three independent measurements. ${ }^{*} p<0.05 ;{ }^{* *} p<0.01$; MOES or MOES MVs vs untreated.

targeted by plant miRNAs and involved in apoptosis and cell proliferation processes. The obtained results showed the presence of mol-miRs, targeting different mRNAs involved in the antiapoptotic mechanisms (Table 5).

Among them, BCL2 transcript was found: BCL2 protein is one of the main factors that regulate tumorigenesis in cell lines of epithelial origin ${ }^{16,35}$ and appear to be the target of both endogenous and exogenous miR$\mathrm{NAs}^{16,35,50,51}$. In our previous works, BCL2 protein expression in lymphoid and monocytoid tumor lines was inhibited by treatment with specific plant miRNAs ${ }^{16}$ and MOES $^{31}$. In both studies, the HD cells (PBMCs) were not sensitive to any type of treatment, underlining the ability of MOES and plant sRNAs to have a specific antitumor activity.

Considering these results, the effect of MOES MVs carrying miRs on the viability and apoptosis mechanisms mediated by BCL2 were analyzed.

MOES MVs had lower toxicity respect to MOES. This difference could be owing to the purification process, which allowed the elimination of the most toxic molecules present in MOES (tannins and alkaloids ${ }^{42,52}$ ).

For this reason, 1 and $5 \mathrm{mg} / \mathrm{ml}$ MOES were sufficient in Jurkat and HeLa cells, respectively, to induce a significant increase in apoptosis and a decrease in cell viability. However, to obtain the same results, the number of MOES MVs contained in 10 or $20 \mathrm{mg} / \mathrm{ml}$ MOES were needed for Jurkat cells and HeLa cells, respectively.

These modifications in viability and apoptosis were associated with a significant decrease in the expression of BCL2 protein.

The permeabilization of the mitochondrial membrane represents a key event for programmed cell death ${ }^{31,53-57}$. BCL2-mediated all treatments caused a significant reduction in mitochondrial membrane potential associated with apoptosis induction.
The observed results demonstrate that MOES MVs, which carry plant miRNAs, can naturally penetrate inside cells and have proapoptotic effects that are comparable to those of MOES but more specific and less toxic.

Environmental exposure and interactions with genetic factors play an important role in human health. In this study, the epigenetic activity carried out by plant-derived miRNAs contained in MOES MVs was demonstrated to regulate human cellular processes at the posttranscriptional level. Moreover, plant miRNAs have been shown to be fundamental bioactive molecules, suggesting the possible use of plant MVs as a natural vehicle to treat humans, even with plant miRNAs.

\section{Materials and methods MOES preparation}

MO mature seeds were harvested in Dschang District, West Cameroon (Africa) by the Cooperative of Medical Plant Producers SOCOPOMO. MO seeds were sun-dried and stored until use. In our laboratory, MOES from MO seed powder was prepared as previously described ${ }^{31}$.

\section{Plant microvesicles purification}

MOES MVs were isolated from known concentrations of MOES, expressed as fresh plant weight (FW) equivalent per $\mathrm{ml}$ of distilled water. The aqueous extract was first filtered by a $0.45-\mu \mathrm{m}$ pore filter, followed by a $0.22 \mu \mathrm{m}$ pore filter, and centrifuged at $13,000 \times g$ for 5 minutes. MOES MVs were stored at $+4{ }^{\circ} \mathrm{C}$ until use.

\section{Plant MVs characterization and quantification}

The MOES MVs were quantified using a Megamix-Plus SSC standard microparticle kit for ILV detection (Biocytex, FRANCE). The Megamix-Plus SSC kit contained nanosized FITC-A-conjugated standardized particles of different sizes $(100-160 \mathrm{~nm}, 160-200 \mathrm{~nm}, 200-240 \mathrm{~nm}$, 240-500 nm). Gates for data acquisition of all vesicle samples were set according to Megamix-Plus SSC nanoparticle standard protocols. The concentration of MOES MVs (number of events $/ \mu \mathrm{l}$ ) was calculated using CytoFLEX (Beckman Coulter, USA), and 150,000 events were acquired in the 100-500 nm range. CytExpert 2.2 software (Beckman Coulter, USA) was used for MOES MVs quantification: in three independent measurements for each of three different MOES MVs purifications, the number of MOES MVs contained in $1 \mathrm{mg}$ (FW) of MOES was $16921 \pm 617$.

\section{Protein, RNA, DNA, and lipid detection in MOES MVs}

For protein extraction, MOES MVs were lysed in RIPA buffer (50 mM Tris- $\mathrm{HCl}, \mathrm{pH}$ 8.0, $150 \mathrm{~mm} \mathrm{NaCl}, 12 \mathrm{~mm}$ deoxycholic acid, $0.5 \%$ Nonidet P-40, and protease and phosphatase inhibitors), and proteins were quantified using the Bradford method. 
Total RNA was extracted from MOES MVs using TRIzol (Life Technologies | Thermo Fisher Scientific-US) according to the manufacturer's protocol.

DNA was extracted from MOES MVs using a NucleoSpin DNA kit (MACHEREY-NAGEL, Germany).

The content of total RNA, DNA, and protein was measured using a NanoDrop Light Spectrophotometer (Thermo Fisher Scientific, USA). In addition to the spectrophotometer measurement method, flow cytometry was used to analyze the presence of RNA, DNA and lipids in MOES MVs using specific probes. For RNA detection, the MOES MVs were labeled with Invitrogen Molecular Probes SYTO RNA Select Green Fluorescent Cell Stain (SYTO RNA) (Thermo Fisher, USA) in accordance with the manufacturer's instructions. The DNA content in MOES MVs was measured using the propidium iodide (PI) method, as described in the Materials and Methods in the Cell Cycle and Apoptosis assays section. For lipid detection, the MOES MVs were stained with BODIPY fluorescent probe (Thermo Fisher Scientific, USA) and incubated at $+4{ }^{\circ} \mathrm{C}$ for 30 minutes.

Stained cells were analyzed using a CytoFLEX flow cytometer (Beckman Coulter, USA) and CytExpert 2.2 software (Beckman Coulter, USA).

The acquisition gate was set according to Megamix-Plus SSC nanoparticle standard protocols as previously described $^{20}$ and reported in Supplementary data.

\section{MOES MVs delivery}

Peripheral blood mononuclear cells (PBMCs) from healthy donors (HDs) and Jurkat and HeLa cell lines were treated with MOES MVs and stained with SYTO RNA, as described above. After 30 minutes of treatment, PBMCs and Jurkat cells were analyzed using CytoFLEX (Beckman Coulter, USA). The HeLa cell line was observed under a LEICA DMI6000B inverted microscope and analyzed using ImageJ software analysis.

\section{M. oleifera small RNA pool extraction and characterization}

The mol-sR pool was extracted from MOES and MOES MVs by a NucleoSpin miRNA kit (MACHEREYNAGEL, Germany). The mol-sR content was determined using a NanoDrop Light Spectrophotometer (Thermo Fisher Scientific, USA), and the mol-miRs contained in the mol-sR pool were characterized through quantitative RT-PCR. The presence and concentration of the most conserved mol-miRs were validated and measured as previously reported ${ }^{16,31,58}$. In detail, a Bio-Rad thermal cycler (IQ5) was used, with the amplification parameters set according to the instructions the EXIQON predesigned primers (Table $\mathrm{S} 1$ ). The relative expression of $\mathrm{mol}$-miRs was quantified by the $2^{-\Delta \Delta \mathrm{Ct}}$ method, where $5 \mathrm{~S}$ RNA was applied as an internal control.

\section{Cell line culture}

Human Jurkat E6-1 lymphoid cells mycoplasma free (American Type Culture Collection, USA) were grown in suspension culture at a density of $0.7 \times 10^{6}$ cells $/ \mathrm{ml}$ in Roswell Park Memorial Institute (RPMI) 1640 medium (Invitrogen, USA).

The HeLa human cervix epithelioid carcinoma cell line, mycoplasma free (American Type Culture Collection, USA) was grown as an adherent monolayer culture at a density of $0.15 \times 10^{6}$ cells $/ \mathrm{ml}$ in Dulbecco's Modified Eagle Medium (Invitrogen, USA). The media of both culture types were supplemented with $10 \%$ fetal bovine serum (fetal bovine serum, Invitrogen, Germany), $2 \mathrm{~mm}$ glutamine (HyClone, $\mathrm{UK}$ ), $50 \mathrm{U} / \mathrm{ml}$ penicillin and $50 \mathrm{U} / \mathrm{ml}$ streptomycin (HyClone, UK).

Human PBMCs from HDs were obtained from 17 individuals attending the local blood transfusion unit of Policlinico 'Tor Vergata' in Rome ${ }^{31}$. PBMCs were separated as previously described ${ }^{59}$. Cell lines and PBMCs were cultured at $37^{\circ} \mathrm{C}$ in a $5 \% \mathrm{CO}_{2}$ humidified atmosphere in the presence or absence of treatment.

\section{Treatment with MOES and MOES MVs}

For the assessment of the cytotoxic effects of MOES and MOES MVs, $0.15 \times 10^{6}$ Jurkat cells/ml, $0.5 \times 10^{5} \mathrm{HeLa}$ cells $/ \mathrm{ml}$ and $1 \times 10^{6} \mathrm{PBMCs} / \mathrm{ml}$ from HDs were treated for $72 \mathrm{~h}$ with MOES at concentrations ranging from $1 \mathrm{mg}$ to $50 \mathrm{mg} / \mathrm{ml}$ of culture medium or MOES MVs extracted from the same concentrations of MOES (number of MVs present in $\mathrm{mg} / \mathrm{ml}$ of MOES). PBMCs were co-stimulated with $20 \mathrm{U} / \mathrm{ml}$ IL2 (Merck KGaD, Germany).

For proliferation and apoptosis studies, Jurkat cells were placed in flasks and treated with $1 \mathrm{mg} / \mathrm{ml}$ MOES or MOES MVs purified from $10 \mathrm{mg}$ MOES for $72 \mathrm{~h}$.

HeLa cells were treated with $5 \mathrm{mg} / \mathrm{ml}$ MOES or MOES MVs purified from $20 \mathrm{mg}$ MOES for $72 \mathrm{~h}$. For the analysis, cells were washed with PBS, harvested, and centrifuged, and the pellets were stored at $-20^{\circ} \mathrm{C}$.

\section{Transfection of the mol-sR pool}

Jurkat and HeLa tumor cells were transfected with mol-sRs at concentrations of $1 \mu \mathrm{g} / \mathrm{ml}$ and $5 \mu \mathrm{g} / \mathrm{ml}$, respectively, for $72 \mathrm{~h}$. Transfection was performed according to the lipofectamine method (Hi-Fect, Qiagen, HF) as previously described ${ }^{16}$.

\section{Cell death/viability assays}

Cell viability and mortality rates were assessed by a $10 \%$ trypan blue (EuroClone S.p.A., Italy) exclusion test after $72 \mathrm{~h}$ of treatment.

\section{Calculation of EC50 and LD50}

Cumulative results from at least three different measurements of three independent experiments performed were used to calculate the concentration of MOES and MOES MVs required to reduce cell viability by $50 \%$ 
(EC50) or to induce death in 50\% of cells (LD50). EC50 and LD50 were evaluated in all cell lines by sigmoidal dose-response regression curves using Graph Pad Prism software as previously described ${ }^{60}$.

\section{Cell cycle and apoptosis assays}

Apoptosis was assessed through flow cytometry analysis of isolated nuclei stained with PI (Sigma) using a CytoFLEX flow cytometer (Beckman Coulter, USA) as previously described ${ }^{31,61,62}$.

In brief, detectors and amplifier gains for forward and orthogonal scatter were adequately selected in order to simultaneously detect nuclei from viable, apoptotic, and necrotic cells. Events were gated on forward versus orthogonal scatter in such a way that degraded DNA from cell debris or from doublets were excluded and nuclei from viable, apoptotic, and necrotic cells were assayed.

Data acquisition and analysis were performed on a minimum of 150,000 events for each sample using CytExpert 2.2 software (Beckman Coulter, USA).

\section{Annexin/PI assay}

Early apoptotic events were detected through double staining of cells with fluorescent annexin V and PI solution. For this purpose, the Annexin V-FITC apoptosis detection kit (BD Biosciences Pharmingen, San José, CA) was used according to the manufacturer's instructions as previously described ${ }^{63}$.

Cells were analyzed immediately after staining by flow cytometry analysis using a CytoFLEX flow cytometer and CytExpert 2.2 software.

\section{Intracellular BCL2 staining}

Intracellular B-cell lymphoma 2 (BCL2) expression was evaluated by flow cytometry analysis. After $72 \mathrm{~h}$, untreated and treated cells were harvested, fixed and permeabilized with 70\% ethanol and incubated with PEconjugated anti-human BCL2 (BD Biosciences, USA). Stained cells were analyzed using CytoFLEX (Beckman Coulter, USA) and CytExpert 2.2 software (Beckman Coulter, USA).

\section{Mitochondrial activity assays JC-1 assay}

To assess the mitochondrial membrane potential in both tumor cell lines, an assay was performed using the MitoProbe JC-1 assay kit for flow cytometry (Molecular Probes Europe BV, Leiden, The Netherlands), as previously described ${ }^{36,53}$. Mitochondrial depolarization is indicated by a decrease in the red/green fluorescence intensity ratio. Cells were analyzed using CytoFLEX (Beckman Coulter, USA) and CytExpert 2.2 software, counting a total of 100,00 events.

\section{Mito Tracker Red FM}

Jurkat and HeLa cells treated with MOES, MOES MVs and the $m o l$-sR pool were stained with Mito Tracker Red FM probe according to the manufacturer's instructions (Thermo Fisher, USA). Red was detected by FL-3 filters. The analysis was assessed using CytoFLEX (Beckman Coulter, USA) and CytExpert 2.2 software, counting a total of 100,00 events in live cells gates.

\section{Bioinformatics analysis}

The mol-miR-human mRNA interactions were studied using a bioinformatics approach as previously described $^{16,20,29}$. Specifically, we investigated the mol-miRhuman mRNA interactions of genes involved in apoptosis.

\section{Statistical analysis}

All data are presented as the mean \pm standard deviation (SD) of at least three independent experiments in triplicate performed on HeLa cells, Jurkat cells and on PBMCs from HDs $(n=17)$. Data analyses were performed using the SPSS statistical software system (version 17.0 for Windows, USA).

Comparisons between treated and untreated cells for the results on the trypan blue assay, apoptosis assay, and BCL2 intracellular protein expression were all conducted using $t$ test. Trypan blue assay results from the different concentrations of extracts and Annexin $\mathrm{V}$ assay were analyzed using analysis of variance (ANOVA) and a Bonferroni significant difference test as a multiple comparison test. Significant differences are shown as * $p<0.05$, *** $p<0.01$, and *** $p<0.001$. For non-parametric correlations, a Pearson correlation coefficient was calculated.

\section{Acknowledgements \\ This project was supported by the STARBIOS2 European Union's Horizon 2020 Research and Innovation Program under grant agreement no. 709517 oriented to promote responsible research and innovation in biosciences. This research was supported by the grant "Consolidate the Foundations 2015", funded by the University of Rome 'Tor Vergata' through the "Progetti finanziati di Ateneo" application, for the project entitled "MIRAGE", grant agreement no. E82F16000610005. The text has been revised for English by Nature Research Editing.}

Patient consent for publication

For the present study, a written statement of consent to participate in the study was obtained from 17 HDs as specified in the Declaration of Helsinki.

\section{Ethical approval}

Ethical approval for the collection and use of human samples was obtained in 2014 from the ethical board of "Tor Vergata" Hospital, protocol number 15/14 (D.M.08.02.2013-D.G.R.146/2013; D.D.G.467 del 25.07.2013). All patients provided written informed consent.

\section{Conflict of interest}

Marina Potestà and Antonella Minutolo received financial support for the grant from the STARBIOS2 European Union's Horizon 2020 Research and Innovation program under grant agreement no. 709517. All authors declare no conflicts of interest with respect to this work. 


\section{Publisher's note}

Springer Nature remains neutral with regard to jurisdictional claims in published maps and institutional affiliations.

The online version of this article (https://doi.org/10.1038/s41420-020-0271-6) contains supplementary material, which is available to authorized users.

Received: 19 December 2019 Revised: 10 April 2020 Accepted: 16 April 2020

Published online: 04 June 2020

\section{References}

1. Rutter, B. D. \& Innes, R. W. Extracellular vesicles as key mediators of plant-microbe interactions. Curr. Opin. Plant Biol. 44, 16-22 (2018).

2. Szempruch, A. J. et al. Extracellular vesicles from trypanosoma brucei mediate virulence factor transfer and cause host anemia. Cell 164, 246-257 (2016).

3. Svennerholm, K. et al. Escherichia coli outer membrane vesicles can contribute to sepsis induced cardiac dysfunction. Sci. Rep. 7, 17434 (2017).

4. Ionescu, M. et al. Xylella fastidiosa outer membrane vesicles modulate plant colonization by blocking attachment to surfaces. Proc. Natl Acad. Sci. USA 111, E3910-E3918 (2014)

5. Hou, Y. et al. A phytophthora effector suppresses trans-kingdom RNAi to promote disease susceptibility. Cell Host Microbe 25, 153-165.e5 (2019).

6. Cui, Y., Gao, J., He, Y. \& Jiang, L. Plant extracellular vesicles. Protoplasma 257, 3-12 (2020).

7. Woith, E. \& Melzig, M. F. Extracellular vesicles from fresh and dried plantssimultaneous purification and visualization using gel electrophoresis. Int. J. Mol. Sci. 20, 357 (2019).

8. Rome, S. Biological properties of plant-derived extracellular vesicles. Food Funct. 10, 529-538 (2019).

9. Woith, E., Fuhrmann, G. \& Melzig, M. F. Extracellular vesicles-connecting kingdoms. International Journal of Molecular Sciences 20, 5695 (2019).

10. Lukasik, A., Brzozowska, I., Zielenkiewicz, U. \& Zielenkiewicz, P. Detection of plant miRNAs abundance in human breast milk. Int. J. Mol. Sci. 19, 37 (2018).

11. Xiao, J. et al. Identification of exosome-like nanoparticle-derived microRNAs from 11 edible fruits and vegetables. PeerJ 5186, 1-19 (2018).

12. $Y u, X$. Odenthal, M. \& Fries, J. W. U. Exosomes as miRNA carriers: formationfunction-future. Int. J. Mol. Sci. 17, 2028 (2016).

13. Zhao, Z., Yu, S., Li, M., Gui, X. \& Li, P. Isolation of exosome-like nanoparticles and analysis of micrornas derived from coconut water based on small RNA highthroughput sequencing. J. Agric. Food Chem. 66, 2749-2757 (2018).

14. Chin, A. R. et al. Cross-kingdom inhibition of breast cancer growth by plant miR159. Cell Res. 26, 217-228 (2016).

15. Hirschi, K. D., Pruss, G. J. \& Vance, V. Dietary delivery: a new avenue for microRNA therapeutics? Trends Biotechnol. 33, 431-432 (2015).

16. Minutolo, A. et al. Olea europaea small RNA with functional homology to human miR34a in cross-kingdom interaction of anti-tumoral response. Sci. Rep. 8, 1-14 (2018).

17. Chen, X., Liang, H., Zhang, J., Zen, K. \& Zhang, C. Y. Secreted microRNAs: a new form of intercellular communication. Trends Cell Biol. 22, 125-132 (2012).

18. Zhang, L. et al. Exogenous plant MIR168a specifically targets mammalian LDLRAP1: evidence of cross-kingdom regulation by microRNA. Cell Res. 22, 107-126 (2012).

19. Zhao, Y., Cong, L. \& Lukiw, W. J. Plant and Animal microRNAs (miRNAs) and Their Potential for Inter-kingdom Communication. Cell Mol. Neurobiol. 38, 133-140 (2018).

20. Aquilano, K. et al. Adipocyte metabolism is improved by TNF receptortargeting small RNAs identified from dried nuts. Commun. Biol. 2, 217 (2019).

21. Li, Z., Xu, R. \& Li, N. MicroRNAs from plants to animals, do they define a new messenger for communication? Nutr. Metab. 15, 68 (2018).

22. Bhattacharya, A., Tiwari, P., Sahu, P. K. \& Kumar, S. A review of the phytochemical and pharmacological characteristics of Moringa oleifera. J. Pharm. Bioall. Sci. 10, 181-191 (2018).

23. Dhongade, H., Kumar, J., Paikra, B. K. \& Gidwani, B. Phytochemistry and pharmacology of Moringa oleifera Lam. J. Pharmacopunct. 20, 194-200 (2017).

24. Kou, X., Li, B., Olayanju, J., Drake, J. \& Chen, N. Nutraceutical or pharmacological potential of Moringa oleifera Lam. Nutrients 10, 343 (2018).

25. Saucedo-Pompa, S. et al. Moringa plants: bioactive compounds and promising applications in food products. Food Res. Int. 111, 438-450 (2018).
26. Cui, J., Zhou, B., Ross, S. A. \& Zempleni, J. Nutrition, microRNAs, and Human Health. Adv. Nutr. Int. Rev. J. 8, 105-112 (2017).

27. Jia, L. et al. Identification of the conserved and novel miRNAs in mulberry by high-throughput sequencing. PLOS ONE 9, e104409.(2014).

28. Jones-Rhoades, M. W. Conservation and divergence in plant microRNAs. Plant Mol. Biol. 80, 3-16 (2012).

29. Pirrò, S. et al. MicroRNA from Moringa oleifera: identification by high throughput sequencing and their potential contribution to Plant medicinal value. PLOS ONE 11, 1-25 (2016).

30. Ju, S. et al. Grape exosome-like nanoparticles induce intestinal stem cells and protect mice from DSS-induced colitis. Mol. Ther. 21, 1345-1357 (2013).

31. Potestà, M. et al. Cytotoxic and apoptotic effects of different extracts of Moringa oleifera Lam on lymphoid and monocytoid cells. Exp. Ther. Med. 18, 5-17 (2019).

32. Teng, Y. et al. Plant-derived exosomal MicroRNAs shape the gut microbiota article plant-derived exosomal MicroRNAs shape the gut microbiota. Cell Host Microbe 24, 1-16 (2018).

33. Matteucci, C. et al. Inhibition of IkBa phosphorylation potentiates regulated cell death induced by azidothymidine in HTLV-1 infected cells. Cell Death Discov. 6, 9 (2020).

34. Mecchia, M. A., Debernardi, J. M., Rodriguez, R. E., Schommer, C. \& Palatnik, J. F. MicroRNA miR396 and RDR6 synergistically regulate leaf development. Mech. Dev. 130, 2-13 (2013).

35. Pirrò, S. et al. Bioinformatics prediction and experimental validation of microRNAs involved in cross-kingdom interaction. J. Comput. Biol. 23, 976-989 (2016).

36. Matteucci, $C$. et al. Characterization of the enhanced apoptotic response to azidothymidine by pharmacological inhibition of NF-kB. Life Sci. 127, 90-97 (2015).

37. Minutolo, A. et al. Apoptosis involves the intrinsic pathway and is p53independent. Cell Death Dis. 3, e358-e359 (2012).

38. Mbikay, M. Therapeutic potential of Moringa oleifera leaves in chronic hyperglycemia and dyslipidemia: a review. Front. Pharmacol. 3, (2012).

39. Suphachai, C. Antioxidant and anticancer activities of Moringa oleifera leaves. J. Med. Plants Res. 8, 318-325 (2014).

40. Vergara-Jimenez, M., Almatrafi, M. \& Fernandez, M. Bioactive components in Moringa oleifera leaves protect against chronic disease. Antioxidants 6, 91 (2017).

41. Fuglie, L. J. Combating malnutrition with Moringa. Development potential for Moringa products (Dar es Salaam, 2001).

42. Zahirah, N., Rani, A., Husain, K. \& Kumolosasi, E. Moringa Genus: a review of phytochemistry and pharmacology. Front. Pharm. 9, 1-26 (2018).

43. Moyo, B., Oyedemi, S., Masika, P. J. \& Muchenje, V. Polyphenolic content and antioxidant properties of Moringa oleifera leaf extracts and enzymatic activity of liver from goats supplemented with Moringa oleifera leaves/sun fl ower seed cake. Meat Sci. 91, 441-447 (2012).

44. Abdull Razis, A. F., Ibrahim, M. D. \& Kntayya, S. B. Health benefits of Moringa oleifera. Asian Pac. J. Cancer Prev. 15, 8571-8576 (2014).

45. Zhou, Z. et al. Honeysuckle-encoded atypical microRNA2911 directly targets influenza A viruses. Cell Res. 25, 39-49 (2015).

46. Xie, W. \& Melzig, M. F. The stability of medicinal plant microRNAs in the herb preparation process. Molecules 2018, 1-12 (2018).

47. Yang, J., Hirschi, K. D. \& Farmer, L. M. Dietary RNAs: new stories regarding oral delivery. Nutrients 7, 3184-3199 (2015).

48. Cekaite, L., Eide, P. W., Lind, G. E. \& Skotheim, R. I. MicroRNAs as growth regulators, their function and biomarker status in colorectal cancer. Oncotarget 7, 6476-6504 (2015)

49. Tafer, H. \& Hofacker, I. L. RNAplex: a fast tool for RNA-RNA interaction search. Bioinformatics 24, 2657-2663 (2008).

50. Conti, B., Minutolo, A., Arciello, M. \& Balsano, C. Are Hedgehog and Wnt/ $\beta$-catenin pathways involved in hepatitis C virus-mediated EMT? J. Hepatol. 58, 636-637 (2013).

51. Chen, J. \& Zhao, K. N. HPV-p53-miR-34a axis in HPV-associated cancers. Ann. Transl. Med. 3, 331 (2015).

52. Wang et al. Proteomic profiles reveal the function of different vegetative tissues of Moringa oleifera. Protein J. 35, 440-447 (2016).

53. Minutolo, A. et al. D(-)lentiginosine-induced apoptosis involves the intrinsic pathway and is p53-independent. Cell Death Dis. 3, e358 (2012).

54. Sarosiek, A. K. \& Triona Ni Chonghaile, A. L. Mitochondria: gatekeepers of response to chemotherapy. Trends Cell Biol. 23, 1-16 (2014). 
55. Poon, I. K. H., Parkes, M. A. F., Jiang, L., Atkin-smith, G. K. \& Tixeira, R. Moving beyond size and phosphatidylserine exposure: evidence for a diversity of apoptotic cell-derived extracellular vesicles in vitro. J. Extracell. Vesicles $\mathbf{8}, 1-14$ (2019).

56. Borutaite, V. Mitochondria as decision-makers in cell death. Environ. Mol. Mutagen. 51, 406-416 (2010)

57. Mignotte, B. \& Vayssiere, J. Mitochondria and apoptosis. Eur. J. Biochem. 252, 1-15 (1998).

58. Gismondi, A., Di Marco, G. \& Canini, A. Detection of plant microRNAs in honey. PLOS ONE 12, 1-12 (2017).

59. Minutolo, A. et al. Lymphocytes as liver damage mirror of HCV related adipogenesis deregulation. 9, 1-10 (2014).
60. Cordero, F. M., Vurchio, C., Macchi, B., Minutolo, A. \& Brandi, A. Synthesis of biotin and fluorescein labeled (-)-lentiginosine. Arch. Organ. Chem. 2014, 215-227 (2014).

61. Kalimutho, M., Minutolo, A., Grelli, S., Federici, G. \& Bernardini, S. Platinum-(IV)derivative satraplatin induced G2/M cell cycle perturbation via p53-p21waf1/ cip1-independent pathway in human colorectal cancer cells. Acta Pharmacol. Sin. 32, 1387-1396 (2011).

62. Cordero, F. et al. -)-(1R,2R,7S,8aR)-1,2,7-Trihydroxyindolizidine[(-)-7S-OH Lentiginosine]: revisited synthesis and disclosure of its proapoptotic activity. Chempluschem 77, 224-233 (2012).

63. Macchi, B. et al. The novel proapoptotic activity of nonnatural enantiomer of Lentiginosine. Glycobiology 20, 500-506 (2010). 